\title{
Ishibashi states, topological orders with boundaries and topological entanglement entropy. Part II. Cutting through the boundary
}

\author{
Ce Shen, ${ }^{c}$ Jiaqi Lou ${ }^{c}$ and Ling-Yan Hung ${ }^{a, b, c, d}$ \\ ${ }^{a}$ State Key Laboratory of Surface Physics, Fudan University, \\ 220 Handan Road, Shanghai 200433, China \\ ${ }^{b}$ Collaborative Innovation Center of Advanced Microstructures, \\ 22 Hankou Road, Nanjing 210093, China \\ ${ }^{c}$ Department of Physics and Center for Field Theory and Particle Physics, Fudan University, \\ 220 Handan Road, Shanghai 200433, China \\ ${ }^{d}$ Institute for Nanoelectronic Devices and Quantum computing, Fudan University, \\ 220 Handan Road, Shanghai 200433, China \\ E-mail: scbebetterme@gmail.com, 17110190002@fudan.edu.cn, \\ elektron.janethung@gmail.com
}

ABStRaCT: We compute the entanglement entropy in a $2+1$ dimensional topological order in the presence of gapped boundaries. Specifically, we consider entanglement cuts that cut through the boundaries. We argue that based on general considerations of the bulkboundary correspondence, the "twisted characters" feature in the Renyi entropy, and the topological entanglement entropy is controlled by a "half-linking number" in direct analogy to the role played by the S-modular matrix in the absence of boundaries. We also construct a class of boundary states based on the half-linking numbers that provides a "closed-string" picture complementing an "open-string" computation of the entanglement entropy. These boundary states do not correspond to diagonal RCFT's in general. These are illustrated in specific Abelian Chern-Simons theories with appropriate boundary conditions.

Keywords: Anyons, Conformal Field Theory, Chern-Simons Theories, Topological Field Theories

ARXIV EPRINT: 1908.07700 


\section{Contents}

1 Introduction 1

2 Entanglement cut across a gapped boundary and twisted character $\quad 2$

2.1 Twisted character in the "open string" frame 3

2.2 Line operators and twisted character in the "closed string" frame 4

$\begin{array}{ll}2.3 & \text { Entanglement entropy from twisted characters }\end{array}$

3 Examples in Abelian Chern-Simons theories $\quad 6$

$\begin{array}{ll}3.1 \text { electric + magnetic b.c } & 7\end{array}$

$\begin{array}{lll}3.2 & \text { electric }+ \text { electric b.c } & 10\end{array}$

3.3 magnetic + magnetic b.c 11

$\begin{array}{ll}3.4 \text { The "closed-string" frame } & 11\end{array}$

3.5 Generic 2-2 K matrix theories, and a condensed-confined duality 14

$\begin{array}{lll}3.6 & \text { A note on the unitarity of } \gamma \text { and more general gapped boundaries } & 17\end{array}$

$\begin{array}{llr}4 \text { Conclusion } & 20\end{array}$

A Setting the notations of Abelian Chern-Simons theories 21

$\begin{array}{lll}\text { A.1 Review of gapped boundaries in } \mathbb{Z}_{N} \text { theory } & 23\end{array}$

B Some useful details of the $D\left(S_{3}\right)$ model $\quad 25$

C Condensed-confined duality of $D\left(S_{3}\right) \quad 25$

$\begin{array}{lr}\text { D } \eta \text { and } \theta \text { functions } & 26\end{array}$

$\begin{array}{ll}\text { E Poisson resummation } & 27\end{array}$

$\begin{array}{lll}\text { F } & \text { Half-linking } \gamma \text { matrix } & 27\end{array}$

$\begin{array}{lll}\text { F.1 } \mathcal{B}=\mathcal{Z}(\mathcal{C}) & 28\end{array}$

F.2 Abelian Chern-Simons 29

$\begin{array}{ll}\text { G TQFT S matrix }=\text { CFT S matrix } & 29\end{array}$

\section{Introduction}

There has been a lot of recent work exploring the effect topological boundaries have on the entanglement entropy of topological phases of matter [1-7]. In our last paper [5] we computed topological entanglement entropies of $2+1$ dimensional topological order using Ishibashi states at the entanglement cut. This paper is a companion paper where we inspect 
the situation where the entanglement cut touches the topological boundaries. This case has been inspected in our previous studies based on lattice models [3], based on recent lattice contructions of topological boundaries [8-12]. It is noticed then that there are non-universal contribution to the topological entanglement at the junction between the entanglement cut and the gapped boundary. Moreover, the basis states naturally selected in the lattice model construction is naturally different from the Ishibashi state construction. In this paper, we argue that based on general considerations of bulk-boundary correspondence, the twisted characters and its modular properties studied also in the CFT literature [13] should control the topological entanglement entropy. The modular transformation of these twisted characters are determined by what we now understand to be the "half-linking" numbers $\gamma_{x c}$. Half-linking numbers are shown to diagonalize the fusion rules of defects [14]. Here, we argue that the topological entanglement entropy is dictated by these $\gamma_{x c}$ when the entanglement cut touches a boundary, in analogy to the role of the modular $S$ matrix in the absence of boundaries [15-17]. We will illustrate these ideas in explicit models based on Abelian Chern-Simons theories. One very important observation, is that the precise treatment at the junction does affect the value of the topological entanglement entropy, as mentioned above. Particularly, we found that there are classes of definition of the twisted characters where the overall normalization of the half-linking number could be altered, giving up unitarity of the matrix. However, there are natural boundary conditions that recover the unitarity of the half-linking matrix.

Our paper is organized as follows. In section 2, we will give general arguments that show how twisted character should feature in the computation of the entanglement entropy of a strip parallel to the axis of a cylinder with two gapped boundaries. We construct the appropriate "Cardy-like — closed string states" that recover the twisted character obtained from edge modes having open-boundaries. In section 3 we illustrate these ideas by computing the entanglement entropy in the case of Abelian Chern-Simons theories. This is computed by quantizing edge modes along the entanglement cut. With appropriate boundary conditions, they recover the twisted character.

We will briefly conclude in section 4 . Some details about Abelian Chern-Simons theories are relegated to the appendix A. For a review of useful facts about gapped boundaries and anyon condensation relevant for the current paper, we refer readers to part I of our paper [5].

\section{Entanglement cut across a gapped boundary and twisted character}

In this section, we would like to consider entangling surfaces that touch or cut through physical boundaries and interfaces. The latter can be obtained from the former via the folding trick. We will therefore focus on the first case. To be concrete, we will consider the entanglement entropy of a strip connecting the top and bottom boundary of a cylinder in Abelian CS theories.

Gapped boundaries are characterized by a Lagrangian algebra $\mathcal{A}$ of a modular tensor category $C$ that describes the bulk topological order in $2+1$ dimensions. In physical terms, the Lagrangian algebra $\mathcal{A}$ corresponds to a set of anyons that condense at the boundary 


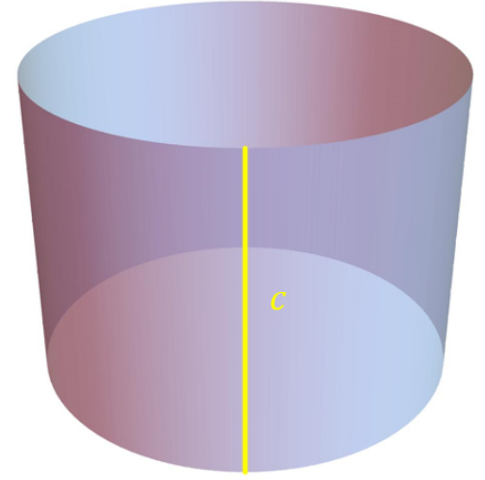

(a) Shared condensed anyon basis.

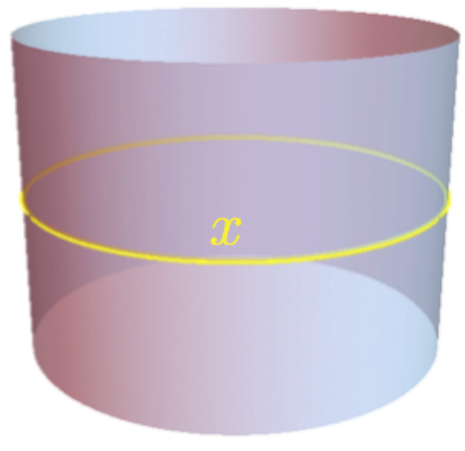

(b) Confined anyon basis.

Figure 1. Ground state basis states on a cylinder.

- they are not conserved across the boundary. This is reviewed in detail in section 2 of our companion paper [5]. We pick up only the issue of ground state basis construction on a cylinder. Consider a cylinder with two boundaries each characterized by an anyon condensate. It generically admits non-trivial ground state degeneracy. There are two ways to construct ground state basis states. One could either consider shared condensed anyon lines connecting the two condensates at the two boundaries. An orthogonal basis carries anyon line that is confined w.r.t. to both condensates that winds the non-contractible cycle of the cylinder. This is illustrated in figure 1.

\subsection{Twisted character in the "open string" frame}

Now consider an entanglement cut that touches the gapped boundary. One could compute the entanglement entropy by taking the entanglement cut as a physical boundary, and determine the Hilbert space on the two sides of the cut. Then we glue the cut back together by constructing an Ishibashi state, corresponding to the fact that the cut is fake and the theory is topological, so that the cut can be arbitrarily deformed. This is the strategy advocated in [17] and adopted also in our previous paper.

In the current situation, the added complication is the physical boundaries that the entanglement cut ends on. There is some appropriate boundary condition at each end, and they are precisely conformal boundary conditions [5]. Now for a given pair of boundary condition $\{x, y\}$, it determines the Hilbert space $H_{x y}$. Like usual open CFT, the Hilbert space $H_{x y}$ admits a decomposition

$$
H_{x y}=\oplus_{z} n_{x y}^{z} H_{z}
$$

where $H_{z}$ corresponds to (left-right) Virasoro representations labeled by $z$. We will see that indeed it gives the set of basis states on a cylinder corresponding to confined anyons winding the non-contractible cycle.

Then the appropriate Ishibashi state that glues the entanglement cut back together should take the form

$$
|\Psi(z)\rangle\rangle=\sqrt{\mathcal{N}} \sum_{k_{L}, k_{R}} \exp \left(-\frac{2 \pi \epsilon}{l} H\right)\left|z, k_{L}, k_{R}\right\rangle_{1} \otimes\left|z, k_{L}, k_{R}\right\rangle_{2},
$$


where $H$ is the Hamiltonian, and $\epsilon \rightarrow 0$ is a regularization parameter, and the product of states is taken between edge states on either side of the entanglement cut. There is a choice of $z$ following from the fact that there is ground state degeneracies on the cylinder.

The trace of the $n$-th-power of the reduced density matrix $\rho$ after tracing out say edge modes labeled 2 , is then given by

$$
\operatorname{tr} \rho^{n}=\mathcal{N}^{n} \chi_{z}(q, \bar{q}), \quad q=\exp (i \tau), \quad \tau=\frac{i 2 \pi \epsilon}{l} .
$$

Recall that a Lagrangian algebra $\mathcal{A}$ is in one-to-one correspondence with modular invariants. Each condensed sector $c_{i} \in \mathcal{A}$ labels a pair of left-right Virasoro representation that features in the modular invariant. i.e.

$$
c_{i} \equiv\left\{h_{L_{i}}, h_{R_{i}}\right\}, \quad \exp \left(i \theta_{c_{i}}\right)=\exp \left(2 \pi i\left(h_{L}-h_{R}\right)\right),
$$

where $\left\{h_{L_{i}}, h_{R_{i}}\right\}$ are the conformal dimensions of the primary operator corresponding to the condensed sector and $\theta_{c_{i}}$ is the topological spin of the sector.

The crucial observation here is that these $x, y, z$ are all labels of line operators in the modular invariant CFT defined by the Lagrangian algebra $\mathcal{A}$ when the two boundaries share the same $\mathcal{A}$. From the perspective of the topological order, these line operators are the confined sectors in the boundary condensate $\mathcal{A}$. The $\chi_{z}$ are twisted characters corresponding to the insertion of these line operators. Some special cases of these line operators and the corresponding twisted characters have been discussed in the CFT literature [13]. Therefore the state (2.2) is the appropriate state describing the confined anyon line $z$ crossing the entanglement cut.

\subsection{Line operators and twisted character in the "closed string" frame}

The twisted character $\chi_{z}$ satisfies

$$
\sum_{z} n_{x y}^{z} \chi_{z}(\tilde{q}, \tilde{\tilde{q}})=\operatorname{tr}_{x \mid y}\left(X_{x} X_{y} q^{-L_{0}} \bar{q}^{-\bar{L}_{0}}\right), \quad \tilde{q} \equiv q^{-1 / \tau},
$$

where $X_{x, y}$ are line operators, and

$$
\chi_{z}(\tilde{q}, \tilde{\bar{q}})=\sum_{i} \gamma_{z c_{i}} \chi_{c_{i}}(q, \bar{q})
$$

where $\gamma_{z c_{i}}$ coincides with the half-linking matrix discussed in [14] and reviewed in the appendix.

The line operators can be written explicitly in the following form

$$
X_{x}=\sum_{c_{i} \in \mathcal{A}} \frac{\gamma_{x c_{i}}}{\sqrt{\gamma_{0 c_{i}}}} \sum_{k_{L}, k_{R}}\left|c_{i}, k_{L}, k_{R}\right\rangle\left\langle c_{i}, k_{L}, k_{R}\right|
$$

where $\left|c_{i}, k_{L}, k_{R}\right\rangle$ are generic descendants at level $\left\{k_{L}, k_{R}\right\}$ of the left-right primary $c_{i}$ inside the Hilbert space defined by the modular invariant characterized by the Lagrangian algebra $\mathcal{A}$. These are analogues of boundary states satisfying the Cardy conditions. Their 
construction in the case of diagonal RCFTs was discussed in [13]. Here, we give a general form using data of the bulk topological order and the half linking matrix $\gamma$.

These line operators $X_{x}$ can in fact be re-arranged into a pair of conformal boundary states via the folding trick, by reversing the bra $\left\langle c_{i}, k_{L}, k_{R}\right|$ into a ket and turning a left moving mode into a right moving mode and vice versa. i.e.

$$
\left.\left.\sum_{k_{L}, k_{R}}\left|c_{i}, k_{L}, k_{R}\right\rangle\left\langle c_{i}, k_{L}, k_{R}\left|\rightarrow \sum_{k_{L}}\right| c_{i}, k_{L}\right\rangle\left|c_{i}, k_{R}^{\prime}=k_{L}\right\rangle \otimes \sum_{k_{R}}\left|c_{i}, k_{R}\right\rangle\left|c_{i}, k_{L}^{\prime}=k_{R}\right\rangle=\left|c_{i}\right\rangle\right\rangle_{L} \otimes\left|c_{i}\right\rangle\right\rangle_{R},
$$

where $\left.\left|c_{i}\right\rangle\right\rangle$ is a conformal boundary state. These conformal boundary states are precisely those basis conformal boundary states that we constructed to describe a condensed anyon ending at the gapped boundary. (See equation (2.15) in the Abelian case and (2.49) for non Abelian ones in part I of our paper [5].) i.e. We can define in place of $X_{x}$,

$$
\left.\left.\left|B_{x}\right\rangle=\sum_{c_{i} \in \mathcal{A}} \frac{\gamma_{x c_{i}}}{\sqrt{\gamma_{0 c_{i}}}}\left|c_{i}\right\rangle\right\rangle_{L} \otimes\left|c_{i}\right\rangle\right\rangle_{R}
$$

Then, equation (2.5) can be re-arranged as overlaps of boundary states $\left|B_{x}\right\rangle$. Their overlaps recover the twisted characters considered above using the analogue of the Cardy condition:

$$
\begin{aligned}
\left\langle B_{z}\left|e^{-H / \delta}\right| B_{y}\right\rangle & =\sum_{c \in \mathcal{C}} \frac{\gamma_{c z}^{\dagger} \gamma_{y c}}{\gamma_{0 c}} \chi_{c}(\tilde{q}) \\
& =\sum_{c, x} \frac{\gamma_{c z}^{\dagger} \gamma_{y c} \gamma_{c x}^{\dagger}}{\gamma_{0 c}} \chi_{x}(q) \\
& =\sum_{x} n_{x z}^{y} \chi_{x}(q)
\end{aligned}
$$

where $n_{x z}^{y}$ is the fusion coefficient of the confined sectors - or equivalently, the line operators of the CFT. We have made use of the defect version of the Verlinde formula found in [14]. We use little $n$ to distinguish the fusion of confined sectors from that of the bulk anyons $N_{a b}^{c}$.

Note that when the two edges of the cylinder are characterized by different anyon condensates, then these line operators $X_{a}$ are interface operators between two different modular invariant CFT's, each determined by the Lagrangian algebra at each end of the cylinder. These twisted characters then transform under modular transformation generically as

$$
\chi_{z}(\tilde{q})=\sum_{c_{i} \in \mathcal{A}_{\mu} \cap \mathcal{A}_{\nu}} \gamma_{z c_{i}}^{(\mu \mid \nu)} \chi_{c_{i}}(q)
$$




\subsection{Entanglement entropy from twisted characters}

The computation of the entanglement entropy of this ground state eigen-basis is thus given by

$$
\begin{aligned}
S_{E E}^{(\mu \mid \nu)}(N \text { strips on a cylinder }) & =2 N\left(\lim _{\substack{n \rightarrow 1 \\
\epsilon \rightarrow 0}} \frac{1}{n-1} \ln \frac{\chi_{x}(\exp (-n \epsilon / l))}{\chi_{x}(\exp (-\epsilon / l))^{n}}\right) \\
& \approx N\left(\lim _{\substack{n \rightarrow 1 \\
\epsilon \rightarrow 0}} \frac{1}{n-1}\left(\ln \frac{\chi_{c_{\min }}(\exp (-l /(n \epsilon)))}{\chi_{c_{\min }}^{n}(\exp (-l / \epsilon))}\right)-\ln \gamma_{x_{c_{\min }}}^{(\mu \mid \nu)}\right)
\end{aligned}
$$

where $\chi_{x}$ are the characters of the Virasoro representation $x$.

The labels $c_{\min }$ denotes the primary corresponding to the shared condensed sectors between boundary $\mu$ and $\nu$, whose conformal dimension is the smallest.

We will illustrate the above in Abelian Chern-Simons theory in the next section. We note that in the case corresponding to $\mathbb{Z}_{N}$ gauge theories, where the electric condensate resides at one boundary and the magnetic condensate the other, we found that the expansion of the character $\chi_{c_{\min }}$ led to a factor of $\sqrt{2}$ following from a non-trivial choice of normalization that records the majorana zero mode, even though $\gamma_{x c}^{(E \mid M)}=1$ there [14].

In the simpler case where both boundaries the entanglement cut touches carry the same set of condensed anyons, $c_{\min }=0$ i.e. $\chi_{c_{\min }}$ is the vacuum Virasoro character, and the above expression reduces to

$$
S_{E E}^{\text {same bc }}(N \text { strips on a cylinder })=2 N\left(\frac{c l}{12 \epsilon}-\ln \gamma_{x 0}\right) .
$$

The area term follows from expanding the vacuum character in the $\epsilon \rightarrow 0$ limit, and the topological entanglement entropy is given simply by $\ln \gamma_{x 0}$, which plays the same role as the modular matrix $S$ in the absence of boundaries.

Consider a bulk phase $B=C \otimes \bar{C}$, where $\bar{C}$ denotes the time-reversal of $C$, and that the boundaries are characterized by a "diagonal" Lagrangian algebra composed of anyons $\{\sigma \otimes \bar{\sigma}\}$ for all $\sigma \in C$. In this case, the cylinder can be unfolded into a torus made of $C$. The entanglement cut can be unfolded into a circle. In this case the confined anyons and condensed anyons can both be labelled by objects in $C$, and it is found that $\gamma_{x c}=S_{x c}^{C}$ [14]. Our result then reduces to the entanglement entropy of $C$ on a closed surface, as expected.

Generic linear combinations of the $x$ eigen-basis leads to a reduced density matrix with superselection sectors labelled by $x$ 's. The probability $p_{x}$ of each sector thus generate an extra classical piece in the entanglement entropy $\Delta S_{E E}=\sum_{x}-p_{x} \ln p_{x}$.

\section{Examples in Abelian Chern-Simons theories}

We consider the case in which a cylinder with gapped boundaries is bi-partitioned into strip regions $R$ and $\bar{R}$. The bulk theory considered here is again the $\mathbb{Z}_{N}$ toric code with action (A.4) and $K$ matrix (A.8). The entanglement entropy between region $R$ and $\bar{R}$ 
is calculated for various boundary conditions in the following. To make things explicit, we will in particular consider the top and bottom physical boundaries $B_{1,2}$ to take the electric/magnetic boundary conditions in turn. Along each of the entanglement cut, we will again recover a pair of edge CFT. For reasons that will become clear later on, we will denote the edge modes on the entanglement cut by $\phi_{I \in\{1,2\}}^{l_{1,2}, r_{1,2}}$. The superscripts $l_{1,2}, r_{1,2}$ refer to the modes on the pair of vertical entanglement cut $b_{1,2}$. Specifically, $l_{i}$ are the degrees of freedom on the left edge of the cut, while $r_{i}$ dofs on the right edge of the cut.

The vertical cut is of length $l$. We will for now work with dimensionless coordinates, and scale it to $\pi$. We will restore dimensions at the end. The physical boundaries $B_{1,2}$ are now located at $x=0, \pi$ respectively. The condensate dictates specific boundary condition that we should impose on the modes $\phi_{I}^{l_{i}, r_{i}}$ at these end points.

In equations (A.23) and (A.24) we presented the conformal boundary conditions that should be satisfied by the edge modes located at the physical boundary, appropriate for the electric and magnetic boundary condensate respectively. In the current situation, these physical boundaries are the end points of the entanglement cut. Therefore the appropriate boundary condition should be replaced by

$$
\left.\sum_{J} l_{J}^{e, m} \partial_{t} \phi_{J}^{l_{i}, r_{i}}\right|_{x=0}=\left.\frac{1}{2 \pi} \partial_{t} \phi_{I}^{l_{i}, r_{i}}\right|_{x=0}=0,
$$

where $l^{e, m}$ are the charge vectors of the condensed anyon characterizing the boundary as reviewed in section A.1. Specifically, here we have $I=1$ for an electric boundary condition and $I=2$ for a magnetic boundary condition. These conditions are satisfied by both the modes on the left/right of the entanglement cut. Similarly boundary condition has to be imposed at $x=\pi$. The problem thus reduces to one of quantizing an "open-string" with specific boundary conditions at the end points (see figure 2a). We will consider combinations of these boundary conditions in turn. From this discussion, it should be clear that the modes that we obtained by quantizing the scalar field with boundary conditions (3.1) correspond to "boundary-changing operator" (bco) that connects the boundaries at the two ends. The topological entanglement entropy then follows from the modular properties of the characters of these bcos.

\section{1 electric + magnetic b.c}

Consider the case where the entanglement cut ends on two different boundaries. We will illustrate this case in detail. In this case the upper $S^{1}$ edge $(x=\pi)$ is electric while the lower $S^{1}$ edge $(x=0)$ is magnetic. From the discussion in [9], we recall that the ground state here is unique. The boundary conditions on the edge modes are

$$
\left\{\begin{array}{l}
\left.\partial_{t} \phi_{2}^{l_{1,2}, r_{1,2}}\right|_{x=0}=0 \\
\left.\partial_{t} \phi_{1}^{l_{1,2}, r_{1,2}}\right|_{x=\pi}=0 .
\end{array}\right.
$$

In terms of the left and right moving chiral fields as in (A.9), we have

$$
\left\{\begin{array}{l}
\left.\partial_{t} \phi_{L}^{l_{1,2}, r_{1,2}}\right|_{x=0}-\left.\partial_{t} \phi_{R}^{l_{1,2}, r_{1,2}}\right|_{x=0}=0 \\
\left.\partial_{t} \phi_{L}^{l_{1,2}, r_{1,2}}\right|_{x=\pi}+\left.\partial_{t} \phi_{R}^{l_{1,2}, r_{1,2}}\right|_{x=\pi}=0 .
\end{array}\right.
$$




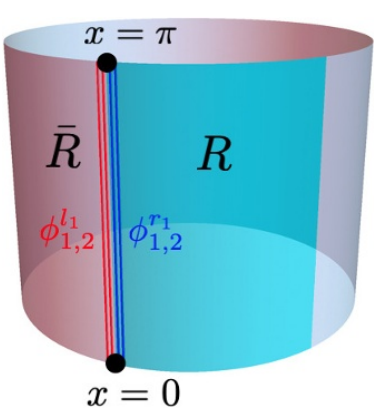

(a) Folded picture.

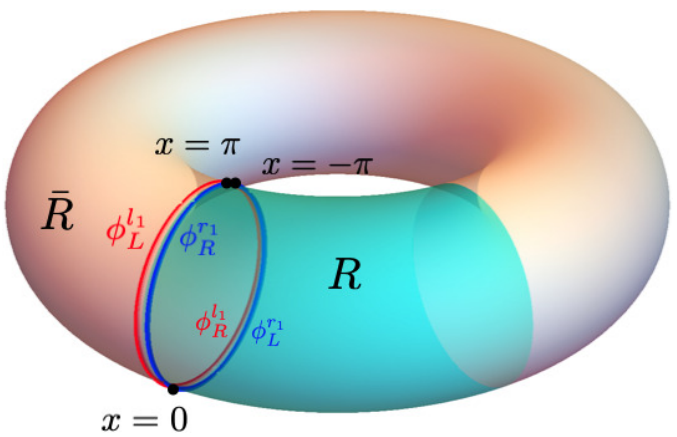

(b) Unfolded picture.

Figure 2. "Open string" modes in the folded picture and "closed string" modes in the unfolded picture.

Since $\phi_{R}(x)$ lives in the interval $(0, \pi)$ and $\phi_{R}(-x)$ lives in the interval $(-\pi, 0)$, we can then simplify notations by combining $\phi_{L, R}^{l_{1,2}, r_{1,2}}$ into "closed-string" fields defined on $(-\pi, 0) \cup(0, \pi)$ (see figure $2 \mathrm{~b}$ ). This gives

$$
\left\{\begin{array}{l}
\Phi_{L}^{1,2}(x)=\phi_{L}^{l_{1,2}}(x) \oplus \phi_{R}^{l_{1,2}}(-x) \\
\Phi_{R}^{1,2}(x)=\phi_{L}^{r_{1,2}}(x) \oplus \phi_{R}^{r_{1,2}}(-x) .
\end{array}\right.
$$

The boundary conditions above translate into continuity and anti-periodicity relations of $\Phi_{L}$

$$
\left\{\begin{array}{c}
\Phi_{L}^{1,2}\left(x=0^{-}\right)=\Phi_{L}^{1,2}\left(x=0^{+}\right) \\
\Phi_{L}^{1,2}(x=-\pi)=-\Phi_{L}^{1,2}(x=\pi) .
\end{array}\right.
$$

Applying the anti-periodicity condition to a general expansion of the chiral mode we obtain

$$
\Phi_{L}^{1,2}=\Phi_{0 L}^{1,2}+2 \pi P_{L}^{1,2} x+i \sum_{n \neq 0} \frac{\alpha_{L, n}^{1,2}}{n} e^{-i n x}
$$

one sees that

$$
\Phi_{0 L}^{1,2}=0, \quad P_{L}^{1,2}=0 .
$$

This corresponds to the fact that we are working with an eigenstate with trivial flux crossing the entanglement cut. This is the only shared confined sector between an electric condensate and a magnetic one.

Also $n$ is a half-integer rather than an integer. Finally the mode expansion becomes

$$
\Phi_{L}^{1,2}=i \sum_{n \in \frac{1}{2}+\mathbb{Z}} \frac{\alpha_{L, n}^{1,2}}{n} e^{-i n x} .
$$

The field $\Phi_{R}^{1,2}$ satisfies exactly the same set of equations, except where we have to take $x \rightarrow-x$ and replace $\alpha_{L}$ by $\alpha_{R}$. 
Each entanglement cut then corresponds to a generalized Ishibashi state matching $\Phi_{L}^{i}$ and $\Phi_{R}^{i}$, in a way completely analogous to the boundary Ishibashi state in [5] except that we have now only half the number of modes. The normalized Ishibashi state at each cut becomes

$$
|0\rangle\rangle_{b_{i}}=e^{-\frac{2 \pi}{2 l} \epsilon H_{i}} \exp \left(\sum_{m \in \frac{1}{2}+\mathbb{N}} \frac{1}{m} \alpha_{-m}^{i} \bar{\alpha}_{-m}^{i}\right)|0\rangle_{b_{i}},
$$

where the Hamiltonian $H_{i}=\left(L_{0}^{i}+\bar{L}_{0}^{i}-1 / 12\right), i$ again denoting the $i$-th entanglement cut. The Hamiltonian is inserted as a UV regularization [17], with the UV cutoff scale given by $\epsilon$. Note that we have restored the dimensionful parameter $l$, the length of the cut, in the expression. There is an extra factor of 2 in $2 l$ since the length of a closed string on the circle doubles that of an open string on the vertical cut.

The normalization constant $\mathcal{N}_{0}$ can be determined by $\langle\langle 0 \mid 0\rangle\rangle=1$,

$$
\begin{aligned}
\mathcal{N}_{0} & =\left\langle 0\left|\exp \left(\sum_{n \in \frac{1}{2}+\mathbb{N}} \frac{1}{n} \alpha_{-n} \bar{\alpha}_{-n}\right) e^{-\frac{4 \pi \epsilon H}{2 l}} \exp \left(\sum_{m \in \frac{1}{2}+\mathbb{N}} \frac{1}{m} \alpha_{-m} \bar{\alpha}_{-m}\right)\right| 0\right\rangle \\
& =q^{-\frac{1}{24}} \sum_{m \in \frac{1}{2} \mathbb{N}} q^{m} \bar{p}(m) \\
& =q^{-\frac{1}{24}} \prod_{r \in \frac{1}{2}+\mathbb{N}} \frac{1}{1-q^{r}} \\
& =q^{-\frac{1}{16}} \sqrt{\frac{\eta(q)}{\theta_{4}(q)}}
\end{aligned}
$$

where $q=e^{\frac{-8 \pi \epsilon}{2 l}}$ and the generating function for degeneracy $\bar{p}(m)$ is given by:

$$
\sum_{m \in \frac{\mathbb{N}}{2}} \bar{p}(m) x^{m}=\prod_{r \in \frac{1}{2}+\mathbb{Z}} \frac{1}{1-x^{r}}
$$

For the trivial topological sector, the lowest eigenstates of $L_{0}$ are listed below

\begin{tabular}{|ccl|}
\hline level $m$ & degeneracy $\bar{p}(m)$ & states \\
\hline 0 & 1 & $|0\rangle$ \\
$\frac{1}{2}$ & 1 & $\alpha_{-\frac{1}{2}}|0\rangle$ \\
1 & 1 & $\alpha_{-\frac{1}{2}}^{2}|0\rangle$ \\
$\frac{3}{2}$ & 2 & $\alpha_{-\frac{1}{2}}^{3}|0\rangle, \alpha_{-\frac{3}{2}}|0\rangle$ \\
\hline
\end{tabular}


Now putting together the two entanglement cuts, the state may be written as a direct product of the two boundaries.

$$
\begin{aligned}
|0\rangle\rangle & \left.=|0\rangle\rangle_{b_{1}} \otimes|0\rangle\right\rangle_{b_{2}} \\
& =e^{-\frac{2 \pi \epsilon H_{1}}{2 l}} \exp \left(\sum_{m \in \frac{1}{2}+\mathbb{N}} \frac{1}{m} \alpha_{-m}^{1} \bar{\alpha}_{-m}^{1}\right)|0\rangle_{b_{1}} \otimes e^{-\frac{2 \pi \epsilon H_{2}}{2 l}} \exp \left(\sum_{n \in \frac{1}{2}+\mathbb{N}} \frac{1}{n} \alpha_{-n}^{2} \bar{\alpha}_{-n}^{2}\right)|0\rangle_{b_{2}} .
\end{aligned}
$$

The reduced density matrix $\rho_{L}$ is obtained by tracing out the anti-chiral part of the full density matrix $\left.\rho=\mathcal{N}_{0}^{-2}|0\rangle\right\rangle\langle\langle 0|$. We therefore have

$$
\begin{aligned}
& \operatorname{Tr}_{L} \rho_{L}^{n}=\left(\frac{\mathcal{N}_{0}\left(q^{n}\right)}{\mathcal{N}_{0}(q)^{n}}\right)^{(a)}\left(\frac{\mathcal{N}_{0}\left(q^{n}\right)}{\mathcal{N}_{0}(q)^{n}}\right)^{(b)} \\
&=\left(\left(\sqrt{\frac{\theta_{4}(q)}{\eta(q)}}\right)^{n} \sqrt{\frac{\eta\left(q^{n}\right)}{\theta_{4}\left(q^{n}\right)}}\right)^{2} \\
&=\left(\left(\sqrt{\frac{\theta_{2}(\tilde{q})}{\eta(\tilde{q})}}\right)^{n} \sqrt{\frac{\eta\left(\tilde{q}^{1 / n}\right)}{\theta_{2}\left(\tilde{q}^{1 / n}\right)}}\right)^{2} \\
& \stackrel{l}{\longrightarrow} \stackrel{\leftrightarrow \rightarrow \infty}{\longrightarrow} 2^{n-1} \tilde{q}^{\frac{1}{12}\left(n-\frac{1}{n}\right)}
\end{aligned}
$$

where in the last step the thermodynamic limit $(l / \epsilon \rightarrow \infty)$ is taken, and the entanglement entropy is

$$
S=\lim _{n \rightarrow 1} \frac{1}{n} \log \operatorname{Tr}_{L} \rho_{L}^{n}=2\left(\frac{\pi l}{12 \epsilon}-\log \sqrt{2}\right) .
$$

We make it explicit with the overall factor of 2 , that the two entanglement cuts of the strip contribute equally to the entanglement entropy. We comment here that the nontrivial topological entanglement entropy $-\log \sqrt{2}$ comes from the two ground states of the orbifold. This is in fact the Majorana mode supposedly trapped at the junction between the electric and magnetic boundaries $[9,14,18,19]$.

\section{2 electric + electric b.c}

In this case the boundary condition becomes

$$
\left\{\begin{array}{l}
\left.\partial_{t} \phi_{1}^{l_{1,2}, r_{1,2}}\right|_{x=0}=0 \\
\left.\partial_{t} \phi_{1}^{l_{1,2}, r_{1,2}}\right|_{x=\pi}=0 .
\end{array}\right.
$$

The analysis is very similar to the previous case, and we will only outline the procedure. The allowed set of zero modes here is given by

$$
P^{1}=0, \quad P^{2}=N a+b,
$$

for all $a \in \mathbb{Z}$, and each $b$ satisfying $0 \leq b \leq N-1$ parametrizes an independent sector. For each fixed $b$, this is an eigenstate of definite flux crossing the entanglement cut. In particular, we note that these are distinct confined sectors relative to the electric condensate at the 
boundaries - i.e. they are the magnetic charges. Ishibashi states can be constructed for each fixed $b$ that entangles the $l, r$ modes at each cut exactly as in the previous subsection, except that the oscillatory modes $\alpha_{n}$ now take integer values of moding $n$. We note that the sum over zero modes contain only a sum over $a$. Alternatively, from the perspective of a combined mode $\Phi_{L, R}$ constructed as in (3.4), it is describable by an effective action characterized by $1 \times 1 K$-matrix $K_{\text {eff }}=(N)$, since the number of edge modes is halved in the presence of boundaries. Then the effective quantum dimension at each entanglement cut is given by $D_{\text {eff }}=\sqrt{N}=\sqrt{D}$, and each entanglement cut contributes $-\ln D_{\text {eff }}=-1 / 2 \ln N$ to the entanglement entropy. (Compare with [5] where each bulk cut contributes $-\ln N$.) Combining the contribution of the two cuts we recover the results in [3].

\section{3 magnetic + magnetic b.c}

The appropriate boundary conditions in this case are given by

$$
\left\{\begin{array}{l}
\left.\partial_{t} \phi_{2}^{l_{1,2}, r_{1,2}}\right|_{x=0}=0 \\
\left.\partial_{t} \phi_{2}^{l_{1,2}, r_{1,2}}\right|_{x=\pi}=0 .
\end{array}\right.
$$

This leads to the following allowed set of zero modes

$$
P^{2}=0, \quad P^{1}=N a+b .
$$

This analysis of this case is equivalent to the electric + electric case. The result of the entanglement entropy is identical. We emphasize again that a natural basis Ishibashi state parametrized by a fixed $b$ corresponds to distinct confined sectors relative to the boundary condensates.

\subsection{The "closed-string" frame}

Written explicitly, the "closed string" electric and magnetic Ishibashi states are respectively given by

$$
\begin{aligned}
\mid a)\rangle_{E} & =\sum_{b \in \mathbb{Z}} \exp \left(-\sum_{l=1}^{\infty} \frac{1}{l} \alpha_{-l} \bar{\alpha}_{-l}\right)\left|P_{L}=P_{R}=\frac{a+b N}{\sqrt{N}}\right\rangle \\
|a\rangle\rangle_{M}= & \sum_{b \in \mathbb{Z}} \exp \left(\sum_{l=1}^{\infty} \frac{1}{l} \alpha_{-l} \bar{\alpha}_{-l}\right)\left|P_{L}=-P_{R}=\frac{a+b N}{\sqrt{N}}\right\rangle
\end{aligned}
$$

where the primary states are orthonormal $\left\langle P_{L}, P_{R} \mid P_{L}^{\prime}, P_{R}^{\prime}\right\rangle=\delta_{P_{L} P_{L}^{\prime}} \delta_{P_{R} P_{R}^{\prime}}$.

In Abelian Chern-Simons theory for example it satisfies

$$
\left.l_{I} \partial_{x} \phi_{I}|c\rangle\right\rangle=0, \quad l \in L,
$$

where $L$ is the set of charge vectors characterizing the gapped boundary condensate as reviewed in (A.16). The subscript $x$ of $\gamma_{x c}$ (as opposed to the spatial coordinate $x$ appearing in $\partial_{x}$ in equation (3.20)) denotes a confined anyon. As demonstrated in the examples above in sections 3.1, 3.2 and 3.3, it is natural to construct eigenbasis so that there is 
definite anyon flux crossing the entanglement cut. The distinct states are thus labeled by sectors confined w.r.t. both condensates at the boundaries of the cylinder. The boundary states in the "closed string" channel are therefore naturally labelled by the same basis. The corresponding "closed-string" boundary states that recover the "open-string" picture satisfying (3.20) is natural in the sense that to convert between the annulus and the cylinder, it is as if we are exchanging $x$ and $t$, and thus replacing the boundary condition (3.1) by (3.20). We note that in the current example,

$$
\gamma_{x c}=\frac{1}{\sqrt{N}} \exp \left(-\frac{2 \pi i c x}{N}\right)=\frac{S_{x c}}{\sqrt{S_{0 c}}} .
$$

In $\mathbf{e}+\mathbf{m}$ case GSD $=1$, no topological sectors remain confined relative to both boundaries, which means every non-contractible Wilson loop can be freely absorbed into the boundaries. Therefore, the only "confined" sector is trivial, $x=0$. So we can omit the confined index $x$ in this case. The overlap of electric and magnetic boundary states projects to the trivial sector:

$$
\begin{aligned}
{ }_{E}\left\langle B\left|e^{-H / \delta}\right| B\right\rangle_{M} & =\frac{1}{\sqrt{N}} E\left\langle\left\langle 0\left|e^{-H / \delta}\right| 0\right\rangle\right\rangle_{M} \\
& =\frac{1}{\sqrt{N}}\left\langle 0\left|\exp \left(-\sum_{l=1}^{\infty} \frac{1}{l} \alpha_{-l} \bar{\alpha}_{-l}\right) e^{-H / \delta} \exp \left(\sum_{l=1}^{\infty} \frac{1}{l} \alpha_{-l} \bar{\alpha}_{-l}\right)\right| 0\right\rangle \\
& =\frac{e^{\frac{\pi}{6 \delta}}}{\sqrt{N}} \prod_{k=1}^{\infty} \frac{1}{1+e^{-4 \pi k / \delta}} \\
& =\frac{\sqrt{2}}{\sqrt{N}} \sqrt{\frac{\eta(\tilde{q})}{\theta_{2}(\tilde{q})}}
\end{aligned}
$$

where $|0\rangle$ denotes the lowest-energy ground state $\left|P_{L}=0, P_{R}=0\right\rangle$ and $\tilde{q}=e^{-4 \pi / \delta}$. Note that the definition of the boundary state $|B\rangle_{E}$ (resp. $|B\rangle_{M}$ ) involves the halflinking matrix $\gamma^{(E \mid E)}$ (resp. $\left.\gamma^{(M \mid M)}\right)$. The factor $\sqrt{\frac{2}{N}}$ here is due to normalization of Ishibashi states [20]. Then the modular transformation of $\eta$ and $\theta_{2}$ function (see appendix D) recovers the open string character (3.10) under the identification $\delta=2 \epsilon / l$.

In $\mathbf{e}+\mathbf{e} / \mathbf{m}+\mathbf{m}$ case $\mathrm{GSD}=\mathrm{N}$, labeled by the electric Wilson lines connecting physical boundaries (condensed sector $c$ ), or altenatively labeled by incontractible magnetic Wilson loops (confined sector $x$ ). The transformation matrix relating the two bases is $\gamma$ that will appear below. The open string quantization (3.15) gives the following character

$$
\begin{aligned}
\chi_{x}(q) & =\sum_{b_{1}, b_{2} \in \mathbb{Z}}\left\langle\frac{x+b_{1} N}{\sqrt{N}}\left|\exp \left( \pm \sum_{l=1}^{\infty} \frac{1}{l} \alpha_{-l} \bar{\alpha}_{-l}\right) e^{\frac{-2 \epsilon \pi}{l} H} \exp \left( \pm \sum_{l=1}^{\infty} \frac{1}{l} \alpha_{-l} \bar{\alpha}_{-l}\right)\right| \frac{x+b_{2} N}{\sqrt{N}}\right\rangle \\
& =\sum_{b \in \mathbb{Z}} \frac{\exp \left(-\pi \delta \frac{(x+b N)^{2}}{2 N}\right)}{\eta(q)}=\frac{1}{\sqrt{N} \eta(\tilde{q})} \sum_{k \in \mathbb{Z}} \exp \left(-\frac{2 \pi}{N \delta} k^{2}-\frac{2 \pi i x}{N} k\right)
\end{aligned}
$$




$$
\begin{aligned}
& =\frac{1}{\sqrt{N} \eta(\tilde{q})} \sum_{n \in \mathbb{Z}} \sum_{c=0}^{N-1} \exp \left(-\frac{2 \pi}{N \delta}(n N+c)^{2}-\frac{2 \pi i c x}{N}\right) \\
& =\sum_{c=0}^{N-1} \gamma_{x c} \chi_{c}(\tilde{q})
\end{aligned}
$$

where $q=e^{-2 \pi \epsilon / l}=e^{-\pi \delta}$ and $\tilde{q}=e^{-4 \pi / \delta}$ under the identification $\delta=2 \epsilon / l$. Poisson resummation is performed in the second line. In the third line we rewrite $k=n N+m$ and split the sum $\sum_{k \in \mathbb{Z}}$ into a double sum $\sum_{n \in \mathbb{Z}} \sum_{c=0}^{N-1}$. As before, $c$ labels different topological sectors condensed at the boundaries, while $n$ labels the primary states corresponding to the same topological sector.

We focus on $\mathbf{e}+\mathbf{e}$ case in the following, since $\mathbf{m}+\mathbf{m}$ case is analyzed in a similar manner. Note that the building block of boundary state overlap, $\left\langle\left\langle c\left|e^{-H / \delta}\right| c\right\rangle\right\rangle$, can be considered as the amplitude of a closed string propagating $1 / \delta$ along the Euclidean time direction, and hence as an open string amplitude via open-closed duality. Explicitly, the building block can be expressed as

$$
\begin{aligned}
& \left\langle\left\langle c\left|e^{-H / \delta}\right| c\right\rangle\right\rangle \\
& =\sum_{n_{1}, n_{2} \in \mathbb{Z}}\left\langle\frac{c+n_{1} N}{\sqrt{N}}\left|\exp \left( \pm \sum_{l=1}^{\infty} \frac{1}{l} \alpha_{-l} \bar{\alpha}_{-l}\right) e^{-H / \delta} \exp \left( \pm \sum_{l=1}^{\infty} \frac{1}{l} \alpha_{-l} \bar{\alpha}_{-l}\right)\right| \frac{c+n_{2} N}{\sqrt{N}}\right\rangle \\
& =\frac{1}{\eta(\tilde{q})} \sum_{n \in \mathbb{Z}} \exp \left(-\frac{4 \pi}{\delta} \frac{(c+n N)^{2}}{2 N}\right) \\
& =\chi_{c}(\tilde{q}) .
\end{aligned}
$$

With the building block at our disposal, the overlap between boundary Cardy states (2.7) is easily identified with open string amplitude (3.23):

$$
\begin{aligned}
\left\langle B_{z}\left|e^{-H / \delta}\right| B_{y}\right\rangle & =\sum_{c \in \mathcal{C}} \frac{\gamma_{c z}^{\dagger} \gamma_{c y}}{\gamma_{0 c}}\left\langle\left\langle c\left|e^{-H / \delta}\right| c\right\rangle\right\rangle \\
& =\sum_{c \in \mathcal{C}} \frac{\gamma_{c z}^{\dagger} \gamma_{c y}}{\gamma_{0 c}} \chi_{c}(\tilde{q}) .
\end{aligned}
$$

More generically, the $\gamma$ matrix for the bulk topological order (A.8) is given by

$$
\gamma_{x c}=\frac{S_{x c}}{\sqrt{S_{0 c}}}=\frac{1}{\sqrt{N}} \exp \left(2 \pi i l_{x}^{T} K^{-1} l_{c}\right)
$$

where we assign to the condensed sector $c$ a charge vector $l_{c} \in L$, and assign to the confined sectors charge vectors $l_{y, z}$. The designation is not unique since any charge vector $l^{\prime}=l+K \Lambda$ with $\Lambda$ an integer vector corresponds to the same topological sector. Here, we have $l_{c}^{T}=(c, 0)$, whereas $l_{y}^{T}=(0, y)$ and $l_{z}^{T}=(0, z)$. If we choose $z-y \equiv x \quad \bmod N$, then $\gamma_{z c}^{\dagger} \gamma_{y c} / \gamma_{0 c}=\gamma_{x c}$ and (3.25) is exactly equal to (3.23). 


\subsection{Generic 2-2 K matrix theories, and a condensed-confined duality}

Consider Abelian topological order given by K matrix, in order that the bulk can support gapped boundary, a necessary but not sufficient condition is that the quadratic form must have total signature 0 , namely there exists a matrix $A$ such that the $\mathrm{K}$ matrix can be diagonalized as follows:

$$
A^{T} K A=\left(\begin{array}{cc}
1 & 0 \\
0 & -1
\end{array}\right) .
$$

In Abelian topological order, the gapped boundary is described by a Lagrangian subgroup $M . M$ is an integer matrix whose column vectors are the condensed anyons, and the group structure is provided by anyon fusion. For convenience we call the set of condensed anyons the condensate, and the set of confined anyons the confinate. The condensate $M$ satisfies self-null and mutual-null condition $M^{T} K^{-1} M=0$. So

$$
M=\left\{\mathbf{m} \in \mathbb{Z}^{2} \mid M^{T} K^{-1} \mathbf{m}=0\right\}=\operatorname{ker}\left(M^{T} K^{-1}\right) \cap \mathbb{Z}^{2}=K \operatorname{ker}\left(M^{T}\right) \cap \mathbb{Z}^{2} .
$$

Given a boundary condensate $M$, the open string touching this boundary has to satisfy the boundary condition (3.1), which is equivalent to $M^{T} A A^{T} N=0$ where $N$ is the confinate $^{1}$ corresponding to the condensate $M$. So

$$
N=\left\{\mathbf{n} \in \mathbb{Z}^{2} \mid M^{T} A A^{T} \mathbf{n}=0\right\}=\operatorname{ker}\left(M^{T} A A^{T}\right) \cap \mathbb{Z}^{2}=\left(A A^{T}\right)^{-1} \operatorname{ker}\left(M^{T}\right) \cap \mathbb{Z}^{2} .
$$

For a cylinder topology with two physical boundaries $M_{\mu}$ and $M_{\nu}$, the set of shared condensed anyons is

$$
\begin{aligned}
M_{\mu} \cap M_{\nu} & =\left\{\mathbf{m} \in \mathbb{Z}^{2} \mid M_{\mu}^{T} K^{-1} \mathbf{m}=0, M_{\nu}^{T} K^{-1} \mathbf{m}=0\right\} \\
& =K \operatorname{ker}\left(M_{\mu}^{T}\right) \cap K \operatorname{ker}\left(M_{\nu}^{T}\right) \cap \mathbb{Z}^{2} \\
& =K\left(\operatorname{ker}\left(M_{\mu}^{T}\right) \cap \operatorname{ker}\left(M_{\nu}^{T}\right)\right) \cap \mathbb{Z}^{2} \\
& =K \operatorname{ker}\left(M_{\mu} M_{\nu}\right)^{T} \cap \mathbb{Z}^{2} \\
& \equiv K \Omega_{\mu \nu} \cap \mathbb{Z}^{2}
\end{aligned}
$$

where we define a line $\Omega_{\mu \nu} \subset \mathbb{R}^{2}$ as $\Omega_{\mu \nu}=\operatorname{ker}\left(M_{\mu} M_{\nu}\right)^{T}$ for convenience. ${ }^{2}$

The cylinder confinate is determined by two sets of boundary conditions (3.1) corresponding to the top $\left(M_{\mu}\right)$ and bottom $\left(M_{\nu}\right)$ boundaries. So the cylinder confinate is

$$
\begin{aligned}
N_{\mu} \cap N_{\nu} & =\left\{\mathbf{n} \in \mathbb{Z}^{2} \mid M_{\mu}^{T} A A^{T} \mathbf{n}=0, M_{\nu}^{T} A A^{T} \mathbf{n}=0\right\} \\
& =\left(A A^{T}\right)^{-1} \operatorname{ker}\left(M_{\mu}^{T}\right) \cap\left(A A^{T}\right)^{-1} \operatorname{ker}\left(M_{\nu}^{T}\right) \cap \mathbb{Z}^{2} \\
& =\left(A A^{T}\right)^{-1}\left(\operatorname{ker}\left(M_{\mu}^{T}\right) \cap \operatorname{ker}\left(M_{\nu}^{T}\right)\right) \cap \mathbb{Z}^{2} \\
& =\left(A A^{T}\right)^{-1} \operatorname{ker}\left(M_{\mu} M_{\nu}\right)^{T} \cap \mathbb{Z}^{2} \\
& =\left(A A^{T}\right)^{-1} \Omega_{\mu \nu} \cap \mathbb{Z}^{2} .
\end{aligned}
$$

\footnotetext{
${ }^{1}$ Here and in the following we assume that the confined sectors have bulk representatives, and we take these bulk representatives as elements of the confinate $N$.

${ }^{2}$ The case $\operatorname{dim} \Omega_{\mu \nu}=0$ is impossible under the assumption that bulk representatives of the confined sectors exist.
} 
On the other hand, by definition the cylinder confinate is composed of the anyons that cannot escape from either boundary. In this sense it is generated by the orthogonal complement of all possible fusion results of the condensed anyons, i.e., $\left(\operatorname{Im}\left(M_{\mu}\right) \cup \operatorname{Im}\left(M_{\nu}\right)\right)^{\perp}$. There's a general relation between the kernel and image of a linear operator, $\operatorname{ker} \mathcal{P}^{T}=$ $(\operatorname{Im} \mathcal{P})^{\perp}$, from which the above expression can be simplified:

$$
\begin{aligned}
N_{\mu} \cap N_{\nu} & =\left(\operatorname{Im}\left(M_{\mu}\right) \cup \operatorname{Im}\left(M_{\nu}\right)\right)^{\perp} \cap \mathbb{Z}^{2} \\
& =\operatorname{Im}\left(M_{\mu}: M_{\nu}\right)^{\perp} \cap \mathbb{Z}^{2} \\
& =\operatorname{ker}\left(M_{\mu}: M_{\nu}\right)^{T} \cap \mathbb{Z}^{2} \\
& =\Omega_{\mu \nu} \cap \mathbb{Z}^{2} .
\end{aligned}
$$

Comparing (3.31) with (3.32) we find that the line $\Omega_{\mu \nu}$ is an invariant subspace of $A A^{T}$ :

$$
A A^{T} \Omega_{\mu \nu}=\Omega_{\mu \nu} .
$$

The self and mutual null conditions of the condensate impose a constraint on the confinfed subspace $\Omega_{\mu \nu}$ :

$$
\Omega_{\mu \nu}^{T} K \Omega_{\mu \nu}=\left(K \Omega_{\mu \nu}\right)^{T} K^{-1}\left(K \Omega_{\mu \nu}\right)=0 .
$$

From (3.27), (3.33) and (3.34) we can also derive

$$
\Omega_{\mu \nu}^{T} K^{-1} \Omega_{\mu \nu}=0 .
$$

It is easily observed here that the "confined direction" $\Omega_{\mu \nu}$ indeed satisfies the equation for "condensed direction" (3.35). For a fixed bulk topological order, the condensate $M$ and confinate $N$ for some boundary (given by boundary condensate $M$ ) become the confinate and condensate respectively for another boundary (given by boundary condensate $N$ ), and vice versa. The roles played by the condensate and the confinate can be swapped. Given an unordered condensate-confinate pair $(M, N)$, it is impossible to distinguish which is which without other information. We call this duality the condensed-confined duality. it is a duality between the condensate and the confinate, or equivalently, between direction $K \Omega_{\mu \nu}$ and $\Omega_{\mu \nu}$.

According to this duality, the relations appeared above (3.33), (3.34), (3.35) still hold under the swapping $\Omega_{\mu \nu} \leftrightarrow K \Omega_{\mu \nu}$. In particular, $A A^{T} K \Omega_{\mu \nu}=K \Omega_{\mu \nu}$ and therefore $K^{2} \Omega_{\mu \nu}=\Omega_{\mu \nu}$.

The condensed-confined duality also appears in the non-Abelian $D\left(S_{3}\right)$ example, we refer the readers to appendix $\mathrm{C}$ for a detailed discussion.

Now we turn to several important 1D lattices appearing in the calculation of open string characters. We define (see figure 4)

$$
\Gamma:=\Omega_{\mu \nu} \cap K \mathbb{Z}^{2}, \quad \tilde{\Gamma}:=K \Omega_{\mu \nu} \cap K \mathbb{Z}^{2},
$$

their dual lattices are respectively given by

$$
\Gamma^{\star}=\Omega_{\mu \nu} \cap K^{-1} \mathbb{Z}^{2}, \quad \tilde{\Gamma}^{\star}=K \Omega_{\mu \nu} \cap K^{-1} \mathbb{Z}^{2},
$$




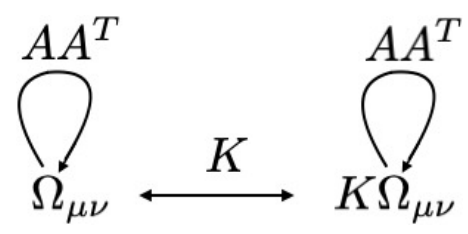

Figure 3. $K \Omega_{\mu \nu}$ and $\Omega_{\mu \nu}$ are invariant subspaces of $A A^{T}$.

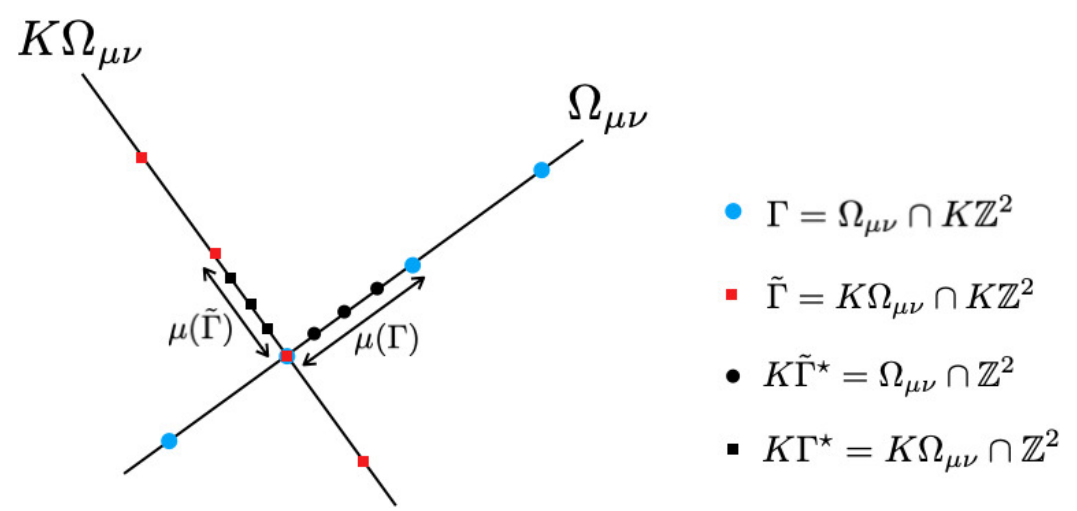

Figure 4. 1D sublattices of the charge lattice $\mathbb{Z}^{2}$.

and satisfying

$$
K \Gamma^{\star}=K \Omega_{\mu \nu} \cap \mathbb{Z}^{2}, \quad K \tilde{\Gamma}^{\star}=\Omega_{\mu \nu} \cap \mathbb{Z}^{2},
$$

we have (see appendix E)

$$
\begin{aligned}
\sum_{\mathbf{m} \in \mathbf{x}+\Gamma} \exp \left(-\pi \mathbf{m}^{T} A A^{T} \mathbf{m}\right) & =\frac{1}{\mu(\Gamma) \sqrt{p(A)}} \sum_{\mathbf{m} \in \Gamma^{\star}} \exp \left(-\pi \mathbf{m}^{T}\left(A A^{T}\right)^{-1} \mathbf{m}+2 \pi i \mathbf{x}^{T} \mathbf{m}\right) \\
& =\frac{1}{\mu(\Gamma) \sqrt{p(A)}} \sum_{\mathbf{n} \in K \Gamma^{\star}} \exp \left(-\pi \mathbf{n}^{T} K^{-1}\left(A A^{T}\right)^{-1} K^{-1} \mathbf{n}+2 \pi i \mathbf{x}^{T} K^{-1} \mathbf{n}\right) \\
& =\frac{1}{\mu(\Gamma) \sqrt{p(A)}} \sum_{c \in \mathcal{C}} \sum_{\mathbf{n} \in \mathbf{c}+\tilde{\Gamma}} \exp \left(-\pi \mathbf{n}^{T} A A^{T} \mathbf{n}+2 \pi i \mathbf{x}^{T} K^{-1} \mathbf{c}\right) \\
& =\frac{1}{\mu(\Gamma) \sqrt{p(A)}} \sum_{\mathbf{c} \in \mathcal{C}} \exp \left(2 \pi i \mathbf{x}^{T} K^{-1} \mathbf{c}\right) \sum_{\mathbf{n} \in \mathbf{c}+\tilde{\Gamma}} \exp \left(-\pi \mathbf{n}^{T} A A^{T} \mathbf{n}\right)
\end{aligned}
$$

where $\mathcal{C}=M_{\mu} \cap M_{\nu}$, and $p(A)$ is the scaling factor (eigenvalue) of the invariant subspace $\Omega_{\mu \nu}$ :

$$
\forall \mathbf{m} \in \Omega_{\mu \nu}, \quad A A^{T} \mathbf{m}=p(A) \mathbf{m},
$$

according to the condensed-confined duality, this factor $p(A)$ is also the scaling factor of the invariant subspace $K \Omega_{\mu \nu}$ (see figure 3 ):

$$
\forall \mathbf{n} \in K \Omega_{\mu \nu}, \quad A A^{T} \mathbf{n}=p(A) \mathbf{n},
$$

and the lattice spacing of $\Gamma$ is equal to the lattice spacing of $\tilde{\Gamma}$ :

$$
\mu(\Gamma)=\mu(\tilde{\Gamma})
$$


so we also have

$$
\begin{aligned}
\sum_{\mathbf{n} \in \mathbf{c}+\tilde{\Gamma}} \exp \left(-\pi \mathbf{n}^{T} A A^{T} \mathbf{n}\right) & =\frac{1}{\mu(\tilde{\Gamma}) \sqrt{p(A)}} \sum_{\mathbf{n} \in \tilde{\Gamma}^{\star}} \exp \left(-\pi \mathbf{n}^{T}\left(A A^{T}\right)^{-1} \mathbf{n}+2 \pi i \mathbf{c}^{T} \mathbf{n}\right) \\
& =\frac{1}{\mu(\tilde{\Gamma}) \sqrt{p(A)}} \sum_{\mathbf{m} \in K \tilde{\Gamma}^{\star}} \exp \left(-\pi \mathbf{m}^{T} K^{-1}\left(A A^{T}\right)^{-1} K^{-1} \mathbf{m}+2 \pi i \mathbf{c}^{T} K^{-1} \mathbf{m}\right) \\
& =\frac{1}{\mu(\tilde{\Gamma}) \sqrt{p(A)}} \sum_{\mathbf{x} \in \mathcal{X}} \sum_{\mathbf{m} \in \mathbf{x}+\Gamma} \exp \left(-\pi \mathbf{m}^{T} A A^{T} \mathbf{m}+2 \pi i \mathbf{c}^{T} K^{-1} \mathbf{x}\right) \\
& =\frac{1}{\mu(\tilde{\Gamma}) \sqrt{p(A)}} \sum_{\mathbf{x} \in \mathcal{X}} \exp \left(2 \pi i \mathbf{c}^{T} K^{-1} \mathbf{x}\right) \sum_{\mathbf{m} \in \mathbf{x}+\Gamma} \exp \left(-\pi \mathbf{m}^{T} A A^{T} \mathbf{m}\right)
\end{aligned}
$$

where $\mathcal{X}=N_{\mu} \cap N_{\nu}$.

(3.39) and (3.43) give rise to the following transformation rules of the open/closed string characters:

$$
\chi_{\mathbf{x}}^{(\text {open })}=\sum_{\mathbf{c} \in \mathcal{C}} \sigma_{\mathbf{x c}} \chi_{\mathbf{c}}^{(\text {closed })}, \quad \chi_{\mathbf{c}}^{(\text {(losed })}=\sum_{\mathbf{x} \in \mathcal{X}} \sigma_{\mathbf{x c}} \chi_{\mathbf{x}}^{(\text {open })}
$$

where

$$
\sigma_{\mathbf{x c}}=\frac{1}{\mu(\tilde{\Gamma}) \sqrt{p(A)}} \exp \left(2 \pi i \mathbf{c}^{T} K^{-1} \mathbf{x}\right)
$$

This non-degenerate square matrix $\sigma$ is unitary (from (3.44)) and satisfies

$$
\frac{\sigma_{\mathrm{xc}}}{\sigma_{0 \mathrm{c}}}=\frac{S_{\mathrm{xc}}}{S_{0 \mathrm{c}}} .
$$

So it is identified with the $\gamma$ matrix: ${ }^{3}$

$$
\sigma_{\mathbf{x c}}=\gamma_{\mathbf{x c}} .
$$

This result guarantees that the topological entanglement entropy where the entanglement cut is cutting across the gapped boundaries characterized by (3.28), (3.29) is given again by (2.12).

\subsection{A note on the unitarity of $\gamma$ and more general gapped boundaries}

In the previous discussions, we demonstrated the explicit construction of boundaries corresponding to different anyon condensation, and how the quantization of the edge modes at the entanglement cut leads to a reduced density matrix whose trace produces the twisted characters. The modular transformation of these twisted characters are determined by a set of half-linking matrix $\gamma$. If one assumes that $\gamma$ is unitary, then it is uniquely determined. In the previous sub-sections, we have presented explicit computations that recover a set of unitary half-linking matrix. We note that there are several choices we have made in our computation, that on hind-sight was responsible for the unitarity of the $\gamma$ matrix.

\footnotetext{
${ }^{3}$ The $\gamma$ matrix for Abelian Chern-Simons theory is explicitly given in [14], from which this relation is easily proved.
} 
Other choices could alter the overall normalization of the gamma matrices, thus shifting the topological entanglement by some non-universal factors.

Specifically, the choice that we have made in the previous calculation is the value of the parameter $r$ that features in the effective action reviewed in equation (A.8). We have chosen $r=1$ in our computation and we note that this is a symmetric point that preserves the symmetry between $\phi_{1}$ and $\phi_{2}$ - which is an electric-magnetic symmetry. While $r$ is canceled out in the computation of the topological entanglement entropy in the absence of boundaries, here it would change the overall normalization of the $\gamma$ matrix and shift the topological entanglement by

$$
\gamma_{x c} \rightarrow \frac{\gamma_{x c}}{\sqrt{\tilde{r}}}, \quad \Delta S_{E E}=-N \ln \tilde{r},
$$

where $N$ is the number of entanglement cuts, and $\tilde{r}$ is the least positive number which makes $r \tilde{r}$ a perfect square.

As a generalization of 3.1 , we consider a cylinder with the bulk given by $\mathbb{Z}_{p q}$ gauge theories and the top and bottom boundaries characterized by subgroups $\mathbb{Z}_{p}$ and $\mathbb{Z}_{q}$ respectively, where $p$ and $q$ are relatively prime and both are greater than 2 [8]. These subgroups specify the subset of magnetic anyons in a Lagrangian algebra, which already uniquely specifies the condensates. The top boundary corresponds to the Lagrangian subgroup $L_{p}=\left\langle\left(\begin{array}{l}p \\ 0\end{array}\right),\left(\begin{array}{l}0 \\ q\end{array}\right)\right\rangle$, while the bottom boundary corresponds to $L_{q}=\left\langle\left(\begin{array}{l}q \\ 0\end{array}\right),\left(\begin{array}{l}0 \\ p\end{array}\right)\right\rangle$. This is a direct generalization of the electric/magnetic boundary conditions considered above. Note that the condensed set $L_{p}$ (or $L_{q}$ ) does not satisfy the mutual null condition - a condition necessary for defining a topological boundary condition for the Chern-Simons theory [21]. To cure the problem, we extend the $2 \times 2 \mathrm{~K}$ matrix to a $4 \times 4$ symmetric integral matrix $\tilde{K}$ by adding 2 one-dimensional edge channels to the boundary [18, 22].

$$
\tilde{K}=K \oplus T=\left(\begin{array}{cccc}
0 & p q & 0 & 0 \\
p q & 0 & 0 & 0 \\
0 & 0 & 0 & 1 \\
0 & 0 & 1 & 0
\end{array}\right)
$$

Adding $T=\left(\begin{array}{ll}0 & 1 \\ 1 & 0\end{array}\right)$ does not introduce any new quasiparticles, so $K$ and $\tilde{K}$ describe the same topological order. The Lagrangian subgroups

$$
L_{p}=\left\langle\left(\begin{array}{l}
p \\
0 \\
0 \\
1
\end{array}\right),\left(\begin{array}{c}
0 \\
q \\
-1 \\
0
\end{array}\right)\right\rangle, \quad L_{q}=\left\langle\left(\begin{array}{l}
q \\
0 \\
1 \\
0
\end{array}\right),\left(\begin{array}{c}
0 \\
p \\
0 \\
-1
\end{array}\right)\right\rangle
$$

are now generated by 4-dimensional charge vectors, satisfying the mutual null condition. For simplicity, we focus on the left side of one particular entanglement cut (superscript $l_{i}$ ) and supress this superscript hereafter. The boundary condition (A.22) becomes

$$
\left\{\begin{array}{l}
\left.\left(p \partial_{t} \phi_{1}+\partial_{t} \phi_{4}\right)\right|_{x=\pi}=0 \\
\left.\left(q \partial_{t} \phi_{2}-\partial_{t} \phi_{3}\right)\right|_{x=\pi}=0 \\
\left.\left(q \partial_{t} \phi_{1}+\partial_{t} \phi_{3}\right)\right|_{x=0}=0 \\
\left.\left(p \partial_{t} \phi_{2}-\partial_{t} \phi_{4}\right)\right|_{x=0}=0 .
\end{array}\right.
$$


We now introduce the 4 left/right moving modes according to decomposition $\tilde{K}=K \oplus T$

$$
\begin{aligned}
\phi_{1} & =\sqrt{\frac{r^{K}}{2 p q}}\left(\phi_{L}^{K}+\phi_{R}^{K}\right), & \phi_{2} & =\frac{1}{\sqrt{2 p q r^{K}}}\left(\phi_{L}^{K}-\phi_{R}^{K}\right), \\
\phi_{3} & =\sqrt{\frac{r^{T}}{2}}\left(\phi_{L}^{T}+\phi_{R}^{T}\right), & \phi_{4} & =\frac{1}{\sqrt{2 r^{T}}}\left(\phi_{L}^{T}-\phi_{R}^{T}\right) .
\end{aligned}
$$

To recover the conformal boundary condition at physical boundaries $x=0$ and $x=\pi$, we demand that (3.51) relates left-moving modes only to right-moving modes. This can only be achieved by tuning parameters to $r^{K}=1, r^{T}=\frac{q}{p}$. Then the boundary condition (3.51) reduces to conformal boundary conditions:

$$
\left\{\begin{array}{l}
\left.\left(\partial_{t} \phi_{L}^{K}-\partial_{t} \phi_{R}^{T}\right)\right|_{x=\pi}=0 \\
\left.\left(\partial_{t} \phi_{R}^{K}+\partial_{t} \phi_{L}^{T}\right)\right|_{x=\pi}=0 \\
\left.\left(\partial_{t} \phi_{L}^{K}+\partial_{t} \phi_{R}^{T}\right)\right|_{x=0}=0 \\
\left.\left(\partial_{t} \phi_{R}^{K}+\partial_{t} \phi_{L}^{T}\right)\right|_{x=0}=0 .
\end{array}\right.
$$

This conformal boundary condition admits only one solution out of all possible values of the zero modes, namely $P_{1}=a, P_{2}=-a, P_{3}=-q a, P_{4}=-p a$, or equivalently $P_{L}^{K}=$ $P_{R}^{T}=0, P_{R}^{K}=-P_{L}^{T}=\sqrt{2 p q} a$ where $a$ is an arbitrary integer. The mode expansion (A.6) together with the conformal boundary condition (3.53) put the following constraint on the excitations:

$$
\left(\begin{array}{cccc}
1 & 0 & 0 & -\lambda \\
0 & \lambda & 1 & 0 \\
1 & 0 & 0 & 1 \\
0 & 1 & 1 & 0
\end{array}\right)\left(\begin{array}{l}
a_{n} \\
b_{n} \\
c_{n} \\
d_{n}
\end{array}\right)=\left(\begin{array}{l}
0 \\
0 \\
0 \\
0
\end{array}\right)
$$

where $\lambda=e^{2 \pi i n}$ and $a_{n}, b_{n}, c_{n}, d_{n}$ are excitations of $\phi_{L}^{K}, \phi_{R}^{K}, \phi_{L}^{T}, \phi_{R}^{T}$ respectively. The determinant of coefficients must vanish in order to have non-trivial excitations, so $(\lambda+$ $1)(\lambda-1)=0$, or $n \in \mathbb{Z} \cup\left\{\frac{1}{2}+\mathbb{Z}\right\}$. (Compare with $\mathbf{e}+\mathbf{m}$ case where $n$ is half-integer, and with $\mathbf{e}+\mathbf{e} / \mathbf{m}+\mathbf{m}$ case where $n$ is integer.)

Following a similar procedure presented in 3.1 we calculate the entanglement entropy between the strip regions $R$ and $\bar{R}$ :

$$
S=2\left(\frac{\pi l}{12 \epsilon}-\log \sqrt{2}-\log \sqrt{2 p q}\right) .
$$

The extension of $K$ matrix and the precise choice of boundary conditions we have chosen in (3.53) introduces a trapped Majorana mode, explaining the term $-2 \log \sqrt{2}$. We see that $r^{T}=\frac{q}{p}$ is a deviation from the symmetric point, leading to a shift $-\log \sqrt{p q}$ in the entanglement entropy. In other words, the half-linking matrix obtained here has a different normalization compared to the unitary one defined in [14].

Finally, one notices that there is one extra $-2 \log \sqrt{2}$ attached alongside $-2 \log \sqrt{p q}$. Physically, this had followed from the fact that we added an extra layer of "topologically 
trivial" material touching the $\mathbb{Z}_{p q}$ through a topological interface described by our boundary conditions (3.51). The interface induces topological symmetry enhancement in the trivial material, so that various trivial sectors in the "trivial material" becomes distinguishable through their connection with the non-trivial anyons. We have discussed this phenomenon already in [5]. The topological entanglement gets contribution from both the $\mathbb{Z}_{p q}$ and $\mathbb{Z}_{1}$ layers, leading to a factor of 2 in front of $p q$.

\section{Conclusion}

In this paper, we demonstrate that the topological entanglement entropy is controlled by the "half-linking" number $\gamma_{x c}$ when the entanglement cut touches the gapped boundaries which are characterized by anyon condensation. We note that when the two gapped boundaries at the end of the entanglement cut correspond to two different anyon condensates, there could potentially be extra contribution to the topological entanglement - as illustrated by the case where the two ends of the entanglement cut touch the electric and magnetic condensates. There is a non-trivial Majorana zero mode that contributes to a factor of $\sqrt{2}$.

One could consider more generic entanglement cuts that cut through gapped interfaces rather than boundaries. However, they could be understood in terms of gapped boundaries using the folding trick.

Our computation was based on an "open-string" quantization at the entanglement cut. We supplement the open-string picture with a "closed-string" picture, by constructing a set of Cardy states suitable also for non-diagonal RCFT's. They are constructed using the half-linking numbers and reproduce the results based on the "open-string" computation. We also prove, at least in the case of Abelian Chern-Simons theories describable by 2-2 $\mathrm{K}$-matrices, that there is a generalized notion of electric-magnetic duality. Namely there is a 1-1 correspondence between condensed and confined anyons and their charge vectors are related by a linear transformation. There is an analogous notion in non-Abelian theories with examples discussed in the appendix, although we could only hope for a precise proof in the future.

We note however, that the normalization of the half-linking matrix can be altered by some subtle change in the edge theory. We show that there is a class of choices which naturally preserve the unitarity of the half-linking matrix. This choice preserves the symmetry between electric and magnetic charges. We also look into other choices of boundaries in which the half-linking matrix has a different overall normalization which departs from the unitary point. They lead to shifts in the normalization which enters into the topological entanglement.

One interesting direction we are currently pursuing is to generalize our work further to cases where the boundary remains gapless. Given the holographic/bulk-boundary correspondence properties found in topological orders which shares many similarities with the AdS/CFT correspondence, the computation should shed some further insight on the Ryu-Takayanagi formula. 


\section{A Setting the notations of Abelian Chern-Simons theories}

The class of Abelian Chern-Simons theories that we are going to consider in the following takes the following form:

$$
S_{\mathrm{CS}}=\frac{1}{4 \pi} \int_{M} K^{I J} A_{I} \wedge F_{J}, \quad F_{J}=d A_{J} .
$$

Where $M$ is a $3 \mathrm{~d}$ manifold. Here $K_{I J}$ is a symmetric integral matrix and $I=1, \ldots, N$. Quantization would involve gauge fixing (such as taking the temporal gauge $A_{t}^{I}=0$ ) and solving for the constraints following from the gauge choice. A review of its detailed procedure can be found for example in [23]. Upon gauge fixing, the action becomes a total derivative. In the temporal gauge for example, the constraint equation would amount to the flat condition

$$
F_{I x y}=0 .
$$

Setting

$$
A_{I x, y}=\partial_{x, y} \phi_{I}
$$

for some scalar function $\phi$ and substituting these expressions into the bulk action, we recover a total derivative term. For $M$ an open manifold with a $2 \mathrm{~d}$ boundary $\partial M$, the total derivative gives rise to the following boundary action

$$
S_{\partial M}=\frac{1}{4 \pi} \int_{\partial M} d t d x\left(K^{I J} \partial_{t} \phi_{I} \partial_{x} \phi_{J}-V^{I J} \partial_{x} \phi_{I} \partial_{x} \phi_{J}\right) .
$$

There is an extra term involving an integral symmetric matrix $V^{I J}$ of rank $m$. As discussed in [24], it is not determined by the bulk CS action. They can be viewed as physical parameters that depend on the actual material supporting these gapless edge modes. We note that $m$ being even is a necessary (although not sufficient) condition for the edge modes to be "gappable" by relevant perturbation. The boundary action can be quantized canonically. This gives, at constant time $t$,

$$
\left[\phi_{I}(x), \Pi^{J}(y)\right]=i \delta_{I}^{J} \delta(x-y), \quad \Pi^{I}(x)=\frac{1}{2 \pi} K^{I J} \partial_{x} \phi_{J} .
$$

Assuming that $x$ is compact and that $x \sim x+l$ i.e. the boundary at constant time $t$ is a ring of length $l$. The mode expansion of $\Phi_{I}$ at $t=0$ is given by

$$
\phi_{I}(x)=\phi_{0 I}+K_{I J}^{-1} P^{J} \frac{2 \pi}{l} x+i \sum_{n \neq 0} \frac{1}{n} a_{I, n} e^{-i n x \frac{2 \pi}{l}} .
$$

These modes therefore satisfy

$$
\left[\alpha_{I, n}, \alpha_{J, m}\right]=n K_{I J}^{-1} \delta_{n,-m},
$$

and for zero modes we have: $\left[\phi_{0 I}, P^{J}\right]=i \delta_{I}^{J}$.

We will focus on the Chern-Simons equivalence of the $D\left(\mathbb{Z}_{N}\right)$ models in the following. The corresponding $K$ matrix is given by

$$
K=\left(\begin{array}{cc}
0 & N \\
N & 0
\end{array}\right) .
$$


The matrix has a pair of eigenvalues with opposite sign, signifying that it has exactly one pair of left and right moving modes, and as such, is a non-chiral theory. The scalars $\phi_{I}$ are related to the left and right moving fields by

$$
\phi_{1}=\sqrt{\frac{r}{2 N}}\left(\phi_{L}+\phi_{R}\right), \quad \phi_{2}=\sqrt{\frac{1}{2 N r}}\left(\phi_{L}-\phi_{R}\right) .
$$

Here $r^{2}=V^{22} / V^{11}$. The left and right moving modes can also be expressed in a mode expansion:

$$
\phi_{L(R)}(x)=\phi_{0 L(R)}+P_{L(R)} \frac{2 \pi}{l} x+i \sum_{n \neq 0} \frac{1}{n} \alpha_{L(R), n} e^{-i n x \frac{2 \pi}{l}} .
$$

To avoid clutter, we will take $r=1$ in the following. We note that $r$ does not play any role in the topological entanglement of a single non-chiral phase. One can show that it is canceled out in the computation of the topological entanglement. It does play a nontrivial role in the discussion of generic interfaces between different $D\left(\mathbb{Z}_{N}\right)$ theories. We will re-introduce them where necessary. We also note that when discussing topological entanglement in a chiral phase, one needs particular care in the choice of $r$. A detailed discussion will be taken up in the accompany paper. In that case, the entanglement cut crosses the physical interfaces, and extra care is needed. Using the commutation relations of $\phi_{I}$, we recover

$$
\left[\alpha_{L(R), n}, \alpha_{L(R), m}\right]=n \delta_{n,-m},
$$

and

$$
P_{L, R}=\frac{1}{\sqrt{2 N}}\left( \pm P^{1}+P^{2}\right)
$$

The U(1)'s gauge groups are taken to be compact. Therefore the scalars are also compact, satisfying

$$
\phi_{I} \sim \phi_{I}+2 \pi
$$

The conjugate momenta to the zero modes therefore are quantized, satisfying

$$
P^{I} \in \mathbb{Z}
$$

We note that these $P_{\phi_{I}}$ parametrizes a set of highest weight states. One can identify these highest weight states/operators with distinct anyons of the quantum double $D\left(\mathbb{Z}_{N}\right)$. The identification with anyons is many-to-one: $P^{I}$ and $P^{I}+N$ describe the same topological sector. One can take $P^{1} \bmod N$ to parametrize the electric charge w.r.t. to the $\mathbb{Z}_{N}$ gauge group in $D\left(\mathbb{Z}_{N}\right)$ models, and $P^{2}$ the magnetic charges. A detailed review can be found in [23]. We only record the basic set of facts needed in the current paper. The Hamiltonian is given by

$$
H=\frac{1}{4 \pi} \int_{0}^{l} d x\left(\partial_{x} \phi_{L} \partial_{x} \phi_{L}+\partial_{x} \phi_{R} \partial_{x} \phi_{R}\right)=\frac{P_{L}^{2}+P_{R}^{2}}{2}+\sum_{n>0}\left(\alpha_{L,-n} \alpha_{L, n}+\alpha_{R,-n} \alpha_{R, n}\right)-\frac{1}{12} .
$$




\section{A.1 Review of gapped boundaries in $\mathbb{Z}_{N}$ theory}

We briefly review gapped boundary and boundary conditions following [5]. Recall that a gapped boundary is characterized by anyon condensation that takes the topological order A to the trivial phase. The set of condensed anyons would form a so called Lagrangian algebra. This has been discussed in general in [18, 21-23, 25-33] and in the special case of Abelian CS theories, in $[21,22]$. In a $D\left(\mathbb{Z}_{N}\right)$ quantum double, all such Lagrangian algebras are known. We can take the set $L$ of condensed anyons as

$$
L=\left\{\left(P^{1}, P^{2}\right)\right\}
$$

where $\left(P^{1}, P^{2}\right)$ is the pair of quantized quantum numbers (see equation (A.9), (A.12)) of the condensed sector.

Among them there are two sets of gapped boundaries that are shared by all $D\left(Z_{N}\right)$ theories and we will take them as examples for illustration purpose. These boundaries are called "electric" and "magnetic" boundaries respectively. Physically, the former correspond to the condensation of all electric charges and magnetic charges respectively i.e.

$$
L_{E}=\{(N n+a, 0)\}, \quad n \in \mathbb{Z}, \quad 0 \leq a \leq N-1,
$$

and similarly

$$
L_{M}=\{(0, N m+b)\}, \quad m \in \mathbb{Z}, \quad 0 \leq b \leq N-1 .
$$

We note that these vectors that are collected into the condensed set $L$ are more selective than picking all charge vectors corresponding to the condensed topological sector. In particular, they are "self-null" and "mutually-null" vectors satisfying

$$
P_{l_{i}}^{I} K_{I J}^{-1} P_{l_{j}}^{J}=0, \quad \forall\left(P_{l_{i}}^{1}, P_{l_{i}}^{2}\right) \in L_{c},
$$

where $L_{c}$ denotes a generic collection of vectors of condensates in a Lagrangian algebra. This has been discussed at length in $[22,23]$, particularly how they are related to existence of corresponding relevant operators that could gap these edge modes.

Alternatively, one can think of these Lagrangian algebra as characterizing conformal boundary conditions $[34,35]$. We note that the boundary theory has a set of U(1) global symmetries extended to a U(1) Kac-Moody algebra. The conserved currents are given by

$$
J_{x}^{I}=\frac{K^{I J}}{2 \pi} \partial_{x} \phi_{J}
$$

This implies that the zero mode of the current is given by

$$
J_{x, 0}^{I} \equiv \int_{0}^{l} d x J_{x}^{I}=P^{I} .
$$

The Lagrangian algebra defines a boundary condition, or alternatively a boundary state $|\psi\rangle\rangle$ that preserves the following symmetries

$$
\left.P_{l_{i}}^{I} K_{I J}^{-1} J_{x}^{J}|\psi\rangle\right\rangle=0 .
$$


Using (A.21), this implies that the boundary condition is allowing the state to carry nontrivial expectation values of $P_{I_{i}}$ simultaneously if they are mutually null, as described in (A.19). Indeed we only need a minimal set of vectors $\left(P_{l_{i}}^{1}, P_{l_{i}}^{2}\right)$ that are linearly independent to generate the entire $L_{c}$. In the case of the electric boundary, we need only the null vector $(1,0)$. i.e.

$$
\left.\left.K_{12}^{-1} J_{x}^{2}|\psi\rangle\right\rangle_{E} \equiv \frac{1}{2 \pi} \partial_{x} \phi_{1}|\psi\rangle\right\rangle_{E}=0 .
$$

Similarly a magnetic boundary would amount to taking the condensate vector $(0,1)$, leading to

$$
\left.\frac{1}{2 \pi} \partial_{x} \phi_{2}|\psi\rangle\right\rangle_{M}=0
$$

Now in terms of the right and left moving fields, the above conditions on the boundary state can be re-written as

$$
\left.\left(J_{L} \pm J_{R}\right)|\psi\rangle\right\rangle_{E / M}=0, \quad J_{L, R}=\frac{1}{2 \pi} \partial_{x} \phi_{L, R},
$$

where $\phi_{L, R}$ are related to $\phi_{1,2}$ by (A.9). We immediately note that the above equations implies that the states $|\psi\rangle\rangle_{E / M}$ are indeed conformal boundary states satisfying the conformal boundary condition,

$$
\left.\left(L_{n}-\bar{L}_{-n}\right)|\psi\rangle\right\rangle_{E / M}=0,
$$

where $L_{n}$ are the Virasoro generators of the left-moving modes and $\bar{L}_{m}$ the corresponding generators of the right-moving modes. This follows from the fact that the stress tensor can be expressed as

$$
T=\pi J_{L} J_{L}, \quad \bar{T}=\pi J_{R} J_{R}
$$

by the Sugawara construction. We note that the Hamiltonian $H$ in (A.15) is indeed given by

$$
H=L_{0}+\bar{L}_{0}-\frac{c}{12}, \quad \text { where } c=1 .
$$

In terms of the mode expansion,

$$
\left.\left(\alpha_{L, n} \pm \alpha_{R,-n}\right)|\psi\rangle\right\rangle_{E / M}=0 .
$$

The corresponding boundary Ishibashi state has the following form:

$$
\left.|\psi\rangle\rangle_{E / M}=\exp \left(-\frac{2 \pi \epsilon}{l} H\right) \exp \left(\mp \sum_{n=1}^{\infty} \frac{1}{n} \alpha_{L,-n} \alpha_{R,-n}\right)\left|P_{L}, P_{R}\right\rangle\right\rangle_{E / M}
$$

where

$$
P_{L}=\mp P_{R},
$$

for electric and magnetic boundaries respectively. The boundary state is not normalizable, and so $\exp \left(-\frac{2 \pi \epsilon}{l} H\right)$ serves as a regularization, with $\epsilon$ infinitesimal. The parameter $l$ is the length of the circle. The norm of this state is then given by

$$
\langle\langle\psi \mid \psi\rangle\rangle=\frac{q^{\frac{P_{L}^{2}}{2}}}{\eta(q)}, \quad q=e^{\frac{-8 \pi \epsilon}{l}} .
$$

This has been discussed for example in [16], although we would like to make the connection to anyon condensation more transparent in the current discussion. 


\section{B Some useful details of the $D\left(S_{3}\right)$ model}

We would like to review here some basic data of the $D\left(S_{3}\right)$ model. The anyons are labeled by $\left(C, \rho_{\alpha_{C}}\right)$, where $C$ is a conjugacy class of the group $G=S_{3}$, and $\alpha_{C}$ an irrep of the centralizer of $C$. A summary of all the anyons are listed below

\begin{tabular}{|c|ccc|cc|cccc|}
\hline & $A$ & $B$ & $C$ & $D$ & $E$ & $F$ & $G$ & $H$ \\
\hline conjugacy class $W$ & & $\{e\}$ & & $\left\{y, x y, x^{2} y\right\}$ & \multicolumn{4}{|c|}{$x, x^{2}$} \\
\hline centralizer $\cong$ & & $S_{3}$ & & & $\mathbb{Z}_{2}$ & \multicolumn{4}{|c|}{$\mathbb{Z}_{3}$} \\
\hline irrep $\rho$ of centralizer & $\mathbf{1}$ & $\operatorname{sign}$ & $\boldsymbol{\pi}$ & $\mathbf{1}$ & $\mathbf{- 1}$ & $\mathbf{1}$ & $\boldsymbol{\omega}$ & $\boldsymbol{\omega}^{*}$ \\
\hline $\operatorname{dim}(\rho)$ & 1 & 1 & 2 & 1 & 1 & 1 & 1 & 1 \\
\hline quantum $\operatorname{dimension} d=|W| \times \operatorname{dim}(\rho)$ & 1 & 1 & 2 & 3 & 3 & 2 & 2 & 2 \\
\hline $\operatorname{twist} \theta$ & 1 & 1 & 1 & 1 & -1 & 1 & $e^{2 \pi i / 3}$ & $e^{-2 \pi i / 3}$ \\
\hline
\end{tabular}

Their fusion rules are given by

\begin{tabular}{|c|ccc|cc|ccc|}
\hline$\otimes$ & $A$ & $B$ & $C$ & $D$ & $E$ & $F$ & $G$ & $H$ \\
\hline$A$ & $A$ & $B$ & $C$ & $D$ & $E$ & $F$ & $G$ & $H$ \\
$B$ & $B$ & $A$ & $C$ & $E$ & $D$ & $F$ & $G$ & $H$ \\
$C$ & $C$ & $C$ & $A \oplus B \oplus C$ & $D \oplus E$ & $D \oplus E$ & $G \oplus H$ & $F \oplus H$ & $F \oplus G$ \\
\hline$D$ & $D$ & $E$ & $D \oplus E$ & $A \oplus C \oplus F \oplus G \oplus H$ & $B \oplus C \oplus F \oplus G \oplus H$ & $D \oplus E$ & $D \oplus E$ & $D \oplus E$ \\
$E$ & $E$ & $D$ & $D \oplus E$ & $B \oplus C \oplus F \oplus G \oplus H$ & $A \oplus C \oplus F \oplus G \oplus H$ & $D \oplus E$ & $D \oplus E$ & $D \oplus E$ \\
\hline$F$ & $F$ & $F$ & $G \oplus H$ & $D \oplus E$ & $D \oplus E$ & $A \oplus B \oplus F$ & $C \oplus H$ & $C \oplus G$ \\
$G$ & $G$ & $G$ & $F \oplus H$ & $D \oplus E$ & $D \oplus E$ & $C \oplus H$ & $A \oplus B \oplus G$ & $C \oplus F$ \\
$H$ & $H$ & $H$ & $F \oplus G$ & $D \oplus E$ & $D \oplus E$ & $C \oplus G$ & $C \oplus F$ & $A \oplus B \oplus H$ \\
\hline
\end{tabular}

The $S$-matrix is given by

$$
S=\frac{1}{6}\left(\begin{array}{cccccccc}
1 & 1 & 2 & 3 & 3 & 2 & 2 & 2 \\
1 & 1 & 2 & -3 & -3 & 2 & 2 & 2 \\
2 & 2 & 4 & 0 & 0 & -2 & -2 & -2 \\
3 & -3 & 0 & 3 & -3 & 0 & 0 & 0 \\
3 & -3 & 0 & -3 & 3 & 0 & 0 & 0 \\
2 & 2 & -2 & 0 & 0 & 4 & -2 & -2 \\
2 & 2 & -2 & 0 & 0 & -2 & -2 & 4 \\
2 & 2 & -2 & 0 & 0 & -2 & 4 & -2
\end{array}\right)
$$

\section{Condensed-confined duality of $D\left(S_{3}\right)$}

There're 4 distinct gapped boundaries for a bulk theory $D\left(S_{3}\right)$, labeled by the 4 different subgroups of $S_{3}$. The condensed anyons corresponding to each subgroup are listed in table 1.

The condensates are not independent. What is previously known is the $C \leftrightarrow F$ duality: a new condensate can be obtained by swapping $C$ and $F$ in a given condensate. We observe here that there's another relation among the condensates. For the gapped boundary 


\begin{tabular}{|c|c|}
\hline subgroup $K$ & condensate \\
\hline $\mathbf{1}$ & $A \oplus B \oplus 2 C$ \\
$\mathbb{Z}_{2}$ & $A \oplus C \oplus D$ \\
$\mathbb{Z}_{3}$ & $A \oplus B \oplus 2 F$ \\
$S_{3}$ & $A \oplus D \oplus F$ \\
\hline
\end{tabular}

Table 1. Condensed anyons corresponding to boundary subgroup $K$.

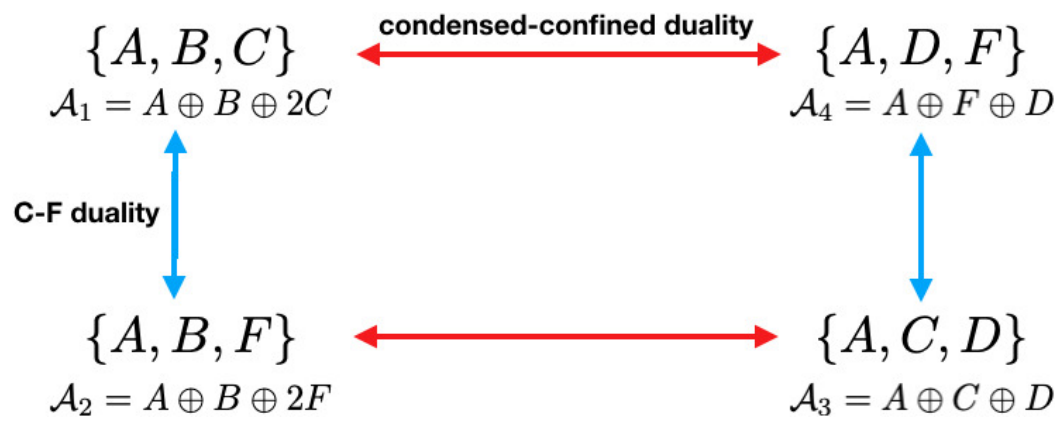

Figure 5. The 4 boundary condensates of $D\left(S_{3}\right)$ Dijkgraaf-Witten model, related by the $C \leftrightarrow F$ duality and the condensed-confined duality.

$\mathcal{A}_{4}=A \oplus D \oplus F$, the confined sectors are labeled by $A, B$ and $C$, which are exactly the condensed anyons for the gapped boundary $\mathcal{A}_{1}=A \oplus B \oplus 2 C$. The situation is more complicated in the inverse direction: for the gapped boundary $\mathcal{A}_{1}=A \oplus B \oplus 2 C$, the confined sectors are labeled by $A, D$ and $F$ with appropriate idempotent splitting [9].

\section{D $\quad \eta$ and $\theta$ functions}

We list here the definitions and basic properties of Dedekind $\eta$-function and Jacobi $\theta$ function.

$$
\begin{aligned}
\eta(\tau) & =q^{\frac{1}{24}} \prod_{n=1}^{\infty}\left(1-q^{n}\right), \\
\theta_{2}(\tau) & =\sum_{n \in \mathbb{Z}} q^{\frac{1}{2}\left(n+\frac{1}{2}\right)^{2}}=2 \eta(\tau) q^{\frac{1}{12}} \prod_{r=1}^{\infty}\left(1+q^{r}\right)^{2}, \\
\theta_{3}(\tau) & =\sum_{n \in \mathbb{Z}} q^{\frac{n^{2}}{2}}=\eta(\tau) q^{-\frac{1}{24}} \prod_{r=0}^{\infty}\left(1+q^{r+\frac{1}{2}}\right)^{2}, \\
\theta_{4}(\tau) & =\sum_{n \in \mathbb{Z}}(-1)^{n} q^{\frac{n^{2}}{2}}=\eta(\tau) q^{-\frac{1}{24}} \prod_{r=0}^{\infty}\left(1-q^{r+\frac{1}{2}}\right)^{2}
\end{aligned}
$$

where $\tau$ is the modular parameter and $q=e^{2 \pi i \tau}$. 
These functions are related by modular $T$ transformation $(\tau \rightarrow \tau+1)$ and $S$ transformation $\left(\tau \rightarrow-\frac{1}{\tau}\right)$ :

$$
\begin{array}{ll}
\eta(\tau+1)=e^{\frac{\pi i}{12}} \eta(\tau), & \eta\left(-\frac{1}{\tau}\right)=\sqrt{-i \tau} \eta(\tau) \\
\theta_{2}(\tau+1)=e^{\frac{\pi i}{4}} \theta_{2}(\tau), & \theta_{2}\left(-\frac{1}{\tau}\right)=\sqrt{-i \tau} \theta_{4}(\tau), \\
\theta_{3}(\tau+1)=\theta_{4}(\tau), & \theta_{3}\left(-\frac{1}{\tau}\right)=\sqrt{-i \tau} \theta_{3}(\tau), \\
\theta_{4}(\tau+1)=\theta_{3}(\tau), & \theta_{4}\left(-\frac{1}{\tau}\right)=\sqrt{-i \tau} \theta_{2}(\tau) .
\end{array}
$$

In the main text $\tilde{q}$ is defined as the $S$ transformation of corresponding $q$, for any complex number $X$

$$
q=e^{X} \stackrel{S}{\rightarrow} \tilde{q}=e^{\frac{4 \pi^{2}}{X}} .
$$

\section{E Poisson resummation}

The Poisson resummation formula is a beautiful relation between a function $f(x)$ and its Fourier transform $\hat{f}(y)=\int e^{2 \pi i\langle x, y\rangle} f(x) d x$, stating that the following infinite sums are equal:

$$
\sum_{n \in \Gamma} f(n)=\frac{1}{\mu(\Gamma)} \sum_{n \in \Gamma^{\star}} \hat{f}(n)
$$

where $\Gamma$ is an $1 \mathrm{D}$ lattice (a free $\mathbb{Z}$-module) and $\mu(\Gamma)$ its lattice spacing (measure of unit cell). $\Gamma^{\star}$ is the dual lattice of $\Gamma$, defined as

$$
\Gamma^{\star}=\{n \in \mathbb{R} \mid\langle m, n\rangle \in \mathbb{Z}, \forall m \in \Gamma\} .
$$

In $1 \mathrm{D}$ the inner product $\langle\cdot, \cdot\rangle$ is reduced to multiplication in $\mathbb{R}$. In particular the following special case is the most frequently invoked in physics literature:

$$
\sum_{n \in \mathbb{Z}} \exp \left(-\pi a n^{2}+b n\right)=\frac{1}{\sqrt{a}} \sum_{k \in \mathbb{Z}} \exp \left(-\frac{\pi}{a}\left(k+\frac{b}{2 \pi i}\right)^{2}\right)
$$

and its inverse

$$
\sum_{k \in \mathbb{Z}} \exp \left(-\pi a(k+b)^{2}\right)=\frac{1}{\sqrt{a}} \sum_{n \in \mathbb{Z}} \exp \left(-\frac{\pi}{a} n^{2}+2 \pi i b n\right) .
$$

The generalization of (E.1) to higher dimension is obvious [36].

\section{F Half-linking $\gamma$ matrix}

We briefly review $\gamma$ matrix following [14]. For a topological order on a cylinder with gapped boundaries $\mu$ and $\nu$, there are naturally two sets of basis to describe the ground 
state subspace, namely the Wilson loop basis $\left|\bigodot_{x}\right\rangle$ labeled by the confined sector $x$, and the Wilson line basis $\left|\uparrow_{c}\right\rangle$ labeled by the condensed sector $c$ shared by the top and bottom physical boundaries. We label by $\mathcal{C}$ the set of shared condensed sectors.

The $\gamma$ matrix is the transformation matrix between the two sets of basis.

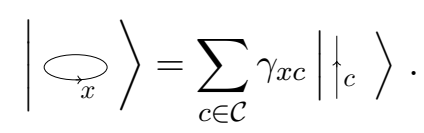

The unitarity of $\gamma$ matrix comes from the orthonormality of the Wilson line basis and the Wilson loop basis:

$$
\begin{aligned}
\delta_{x y} & =\left\langle\bigodot_{x} \mid \bigodot_{y}\right\rangle \\
& =\sum_{c, d}\left\langle\hat{f}_{d}\left|\gamma_{d y}^{\dagger} \gamma_{x c}\right| \dagger_{c}\right\rangle \\
& =\sum_{c, d} \gamma_{d y}^{\dagger} \gamma_{x c} \delta_{c d}=\sum_{c} \gamma_{c y}^{\dagger} \gamma_{x c} .
\end{aligned}
$$

From the unitarity we can obtain the inverse transformation:

$$
\left|\hat{f}_{c}\right\rangle=\sum_{x} \gamma_{c x}^{\dagger}\left|\bigodot_{x}\right\rangle=\sum_{x} \overline{\gamma_{x c}}\left|\bigodot_{x}\right\rangle .
$$

\section{F.1 $\mathcal{B}=\mathcal{Z}(\mathcal{C})$}

If the bulk theory $\mathcal{B}=\mathcal{Z}(\mathcal{C})$ can be factorized $\mathcal{B}=\mathcal{C} \otimes \overline{\mathcal{C}}$ where $\mathcal{C}$ is also a modular tensor category with modular matrix $S^{\mathcal{C}}$, then $\gamma_{x c}=S_{x, c}^{\mathcal{C}}=\frac{S_{x \overline{0}, c \bar{c}}^{\mathcal{B}}}{\sqrt{S_{0 \overline{0}}^{\mathcal{B}}, c \bar{c}}}$.

We can perform the folding/unfolding trick if $\mathcal{B}$ can be factorized. Unfolding the cylinder to a torus, the doubled theory $\mathcal{B}$ splits into $\mathcal{C}$ and $\overline{\mathcal{C}}$. The Wilson loop $\bigodot_{x}$ unfolds to $\bigodot_{x}$ and $\bigodot_{0}$, while the Wilson line $\uparrow_{c}$ unfolds to $\uparrow_{c}$ and $\uparrow_{\bar{c}}$.

The $S^{\mathcal{B}}$ for the doubled theory is the tensor product $S^{\mathcal{B}}=S^{\mathcal{C}} \otimes S^{\overline{\mathcal{C}}}$, so

$$
S_{i \bar{j}, k \bar{l}}^{\mathcal{B}}=S_{i k}^{\mathcal{C}} S_{\bar{j} \bar{l}}^{\overline{\mathcal{C}}}=S_{i k}^{\mathcal{C}} S_{j l}^{\mathcal{C}} .
$$

The last step comes from the fact $S_{\bar{j} \bar{l}}^{\bar{c}}=S_{j l}^{\mathcal{C}}$. Hence we have

$$
\frac{S_{x \overline{0}, c \bar{c}}^{\mathcal{B}}}{\sqrt{S_{0 \overline{0}, c \bar{c}}^{\mathcal{B}}}}=S_{x, c}^{\mathcal{C}}
$$

The $\gamma$ matrix can be identified with the $S^{\mathcal{C}}$ matrix in this case. Because the cylinder

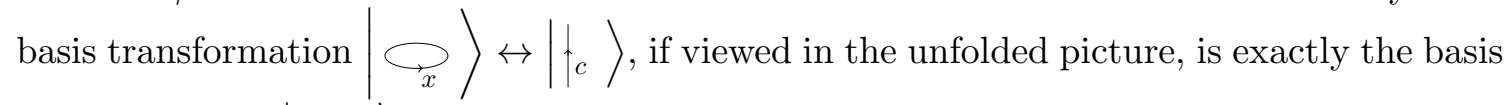
transformation $\left|\ominus_{x}\right\rangle \leftrightarrow\left|\mathcal{G}_{c}\right\rangle$ on a torus, which is dictated by $S^{\mathcal{C}}$ matrix. 


\section{F.2 Abelian Chern-Simons}

If the bulk theory is Abelian Chern-Simons theory given by the $K$ matrix, and both boundaries of the cylinder are described by condensate $C$, then $\gamma_{x c}=\frac{S_{x c}}{\sqrt{S_{0 c}}}$.

$$
\left.\left|\bigoplus_{0}\right\rangle=\frac{1}{\sqrt{D}} \sum_{c \in \mathcal{C}}||_{c}\right\rangle
$$

where the normalization constant $\frac{1}{\sqrt{D}}$ comes from $|\mathcal{C}|=D$. Applying Wilson loop operators to this state

$$
\begin{aligned}
\left|\vec{x}_{x}\right\rangle=\widehat{Q_{x}}\left|Q_{0}\right\rangle & \left.=\frac{1}{\sqrt{D}} \sum_{c \in \mathcal{C}} \widehat{P_{x}}||_{c}\right\rangle \\
& \left.=\frac{1}{\sqrt{D}} \sum_{c \in \mathcal{C}} \frac{S_{x c}}{S_{0 c}}||_{c}\right\rangle \\
& \left.=\frac{S_{x c}}{\sqrt{S_{0 c}}}||_{c}\right\rangle
\end{aligned}
$$

in the last line we've used $S_{0 a}=\frac{d_{a}}{D}=\frac{1}{D}$ for abelian Chern-Simons theory.

\section{G TQFT S matrix $=$ CFT S matrix}

For Abelian Chern-Simons theory on a torus, the TQFT $S$ matrix from anyon braiding is identified with CFT $S$ matrix from modular transformation of characters.

If an Abelian Chern-Simons theory supports gapped edge, then its bulk $K$ matrix must have total signature 0 , and hence congruent to $\left(\begin{array}{cc}1 & 0 \\ 0 & -1\end{array}\right)$. Suppose

$$
A^{T} K A=\left(\begin{array}{cc}
1 & 0 \\
0 & -1
\end{array}\right),
$$

taking the determinant of both sides gives $\operatorname{det} A=\frac{1}{\sqrt{|\operatorname{det} K|}}=\frac{1}{D}$, where $D$ is the total quantum dimension. In abelian Chern-Simons theory, each anyon has quantum dimension 1 , so $D^{2}=\#($ anyon types $)=|\operatorname{det} K|$. The quantized conjugate momentum is: ${ }^{4}$

$$
\left(\begin{array}{c}
P_{1} \\
P_{2}
\end{array}\right)=K^{-1} \mathbf{m}
$$

where $\mathbf{m}=\left(P^{1}, P^{2}\right)^{T}$ is an integer vector. Transforming to the left/right-moving frame,

$$
\left(\begin{array}{l}
P_{L} \\
P_{R}
\end{array}\right)=A^{-1}\left(\begin{array}{l}
P_{1} \\
P_{2}
\end{array}\right)=(K A)^{-1} \mathbf{m} .
$$

\footnotetext{
${ }^{4}$ For simplicity and without loss of generality, we will assume $K$ to be a $2 \times 2$ matrix, the generalization is obvious.
} 
Whenever we want to calculate the character of a CFT living on an entanglement cut, the pattern $\sum e^{-2 \pi H}$ always appear. If we analyze the Hamiltonian closely,

$$
H=\frac{P_{L}^{2}+P_{R}^{2}}{2}+\sum_{n>0}\left(\alpha_{L,-n} \alpha_{L, n}+\alpha_{R,-n} \alpha_{R, n}\right)-\frac{1}{12}
$$

the first part $\frac{P_{L}^{2}+P_{R}^{2}}{2}$ is responsible for Poisson resummation and yields Jacobi $\theta$ function, while the second part $\sum_{n>0}\left(\alpha_{L,-n} \alpha_{L, n}+\alpha_{R,-n} \alpha_{R, n}\right)-\frac{1}{12}$ always gives Dedekind $\eta$ function. The summation in $\sum e^{-2 \pi H}$ is performed over all primaries inside a superselection sector, which is reduced to summation over some integer lattice sites equivalent under $K$. Denote this lattice by $\Gamma$, it is a sublattice of $\mathbb{Z}^{2}$, and satisfies

$$
\forall \mathbf{x}, \mathbf{y} \in \Gamma, \exists \mathbf{n} \in \mathbb{Z}^{2} \text { such that } \mathbf{x}-\mathbf{y}=K \mathbf{n} .
$$

The first part of the Hamiltonian (G.4) can be written as

$$
\begin{aligned}
\frac{1}{2}\left(P_{L}^{2}+P_{R}^{2}\right) & =\frac{1}{2}\left(\begin{array}{ll}
P_{L} & P_{R}
\end{array}\right)\left(\begin{array}{ll}
1 & 0 \\
0 & 1
\end{array}\right)\left(\begin{array}{l}
P_{L} \\
P_{R}
\end{array}\right) \\
& =\frac{1}{2} \mathbf{m}^{T}\left((K A)^{-1}\right)^{T}\left(\begin{array}{ll}
1 & 0 \\
0 & 1
\end{array}\right)(K A)^{-1} \mathbf{m} \\
& =\frac{1}{2} \mathbf{m}^{T} A A^{T} \mathbf{m} .
\end{aligned}
$$

The 1D Poisson resummation formula

$$
\sum_{n \in \mathbb{Z}} \exp \left(-\pi a n^{2}+b n\right)=\frac{1}{\sqrt{a}} \sum_{k \in \mathbb{Z}} \exp \left(-\frac{\pi}{a}\left(k+\frac{b}{2 \pi i}\right)^{2}\right)
$$

can be easily generalized to $2 \mathrm{D}$ :

$$
\sum_{\mathbf{n} \in \mathbb{Z}^{2}} \exp \left(-\pi \mathbf{n}^{T} A \mathbf{n}+\mathbf{b}^{T} \mathbf{n}\right)=\frac{1}{\sqrt{\operatorname{det} A}} \sum_{\mathbf{k} \in \mathbb{Z}^{2}} \exp \left(-\pi\left(\mathbf{k}+\frac{\mathbf{b}}{2 \pi i}\right)^{T} A^{-1}\left(\mathbf{k}+\frac{\mathbf{b}}{2 \pi i}\right)\right) .
$$

Consider $\Gamma_{\mathbf{a}}=K \mathbb{Z}^{2}+\mathbf{a}$, let's focus on the Poisson resummation part of $\exp (-2 \pi H)$

$$
\begin{aligned}
& \sum_{\mathbf{m} \in \Gamma_{a}} \exp \left(-\pi \mathbf{m}^{T} A A^{T} \mathbf{m}\right) \\
= & \sum_{\mathbf{n} \in \mathbb{Z}^{2}} \exp \left(-\pi(K \mathbf{n}+\mathbf{a})^{T} A A^{T}(K \mathbf{n}+\mathbf{a})\right) \\
= & \sum_{\mathbf{n} \in \mathbb{Z}^{2}} \exp \left(-\pi \mathbf{n}^{T} A^{-T} A^{-1} \mathbf{n}-2 \pi \mathbf{a}^{T} A A^{T} K \mathbf{n}-\pi \mathbf{a}^{T} A A^{T} \mathbf{a}\right) \\
= & \frac{1}{\sqrt{\operatorname{det}\left(A^{-T} A^{-1}\right)}} \sum_{\mathbf{k} \in \mathbb{Z}^{2}} \exp \left(-\pi\left(\mathbf{k}+i K A A^{T} \mathbf{a}\right)^{T} A A^{T}\left(\mathbf{k}+i K A A^{T} \mathbf{a}\right)-\pi \mathbf{a}^{T} A A^{T} \mathbf{a}\right) \\
= & \operatorname{det}(A) \sum_{\mathbf{k} \in \mathbb{Z}^{2}} \exp \left(-\pi \mathbf{k}^{T} A A^{T} \mathbf{k}-2 \pi i \mathbf{a}^{T} A A^{T} K A A^{T} \mathbf{k}\right)
\end{aligned}
$$


where in the second line we rewrite $\mathbf{m}=K \mathbf{n}+\mathbf{a}$ and transform the sum over $\mathbf{m}$ to the sum over $\mathbf{n}$. As demonstrated above, the next key step is to split the sum $\sum_{\mathbf{k} \in \mathbb{Z}^{2}}$ to a double sum. To this end, we rewrite $\mathbf{k}=K \mathbf{p}+\mathbf{b}$. In order to recover the $\mathbb{Z}^{2}$ lattice over which $\mathbf{k}$ is summed, $\mathbf{b}$ must run through all possible anyon types, namely $\sum_{\mathbf{k} \in \mathbb{Z}^{2}}=\sum_{\mathbf{p} \in \mathbb{Z}^{2}} \sum_{\mathbf{b} \in L}$. Furthermore $A A^{T} K A A^{T} K=\mathbf{1}$ by the definition of $A$.

$$
\begin{aligned}
& \sum_{\mathbf{m} \in \Gamma_{a}} \exp \left(-\pi \mathbf{m}^{T} A A^{T} \mathbf{m}\right) \\
= & \operatorname{det}(A) \sum_{\mathbf{b} \in L} \exp \left(-2 \pi i \mathbf{a}^{T} K^{-1} \mathbf{b}\right) \sum_{\mathbf{p} \in \mathbb{Z}^{2}} \exp \left(-\pi(K \mathbf{p}+\mathbf{b})^{T} A A^{T}(K \mathbf{p}+\mathbf{b})\right) \\
= & \sum_{\mathbf{b} \in L} \frac{1}{D} \exp \left(-2 \pi i \mathbf{a}^{T} K^{-1} \mathbf{b}\right) \sum_{\mathbf{m} \in \Gamma_{\mathbf{b}}} \exp \left(-\pi \mathbf{m}^{T} A A^{T} \mathbf{m}\right) \\
= & \sum_{\mathbf{b} \in L} S_{\mathbf{a b}} \sum_{\mathbf{m} \in \Gamma_{\mathbf{b}}} \exp \left(-\pi \mathbf{m}^{T} A A^{T} \mathbf{m}\right),
\end{aligned}
$$

where $S_{\mathbf{a b}}=\frac{1}{D} \exp \left(-2 \pi i \mathbf{a}^{T} K^{-1} \mathbf{b}\right)$ is the TQFT $S$ matrix defined as the Hopf link with the two cycles labeled by $a$ and $b$ respectively. Restore the full character by completing the $\eta$ function part and the appropriate modular parameter we get

$$
\chi_{\mathbf{a}}(q)=\sum_{\mathbf{b} \in L} S_{\mathbf{a b}} \chi_{\mathbf{b}}(\tilde{q}) .
$$

Thus we have shown explicitly that the modular transformation of characters is effected by the TQFT $S$ matrix in Abelian Chern-Simons theory.

\section{Acknowledgments}

We thank Laurent Freidel, Gabriel Wong and Yang Zhou for helpful discussions. We would also like to thank Juven Wang for many past conversations on the subject. We are particularly grateful to Yidun Wan for a critical reading of our draft. Part of this work is done during LYH and CS's visit to Perimeter Institute, as part of the EmmyNoether Fellowship programme. LYH acknowledges the support of Fudan University and the Thousands Young Talents Program. This work is supported by the NSFC grant number 11875111.

Open Access. This article is distributed under the terms of the Creative Commons Attribution License (CC-BY 4.0), which permits any use, distribution and reproduction in any medium, provided the original author(s) and source are credited.

\section{References}

[1] J.C. Wang et al., Tunneling Topological Vacua via Extended Operators: (Spin-)TQFT Spectra and Boundary Deconfinement in Various Dimensions, Prog. Theor. Exp. Phys. 2018 (2018) 053A01 [arXiv:1801.05416] [INSPIRE].

[2] B. Shi and Y.-M. Lu, Characterizing topological order by the information convex, Phys. Rev. B 99 (2019) 035112 [arXiv:1801.01519] [INSPIRE]. 
[3] C. Chen, L.-Y. Hung, Y. Li and Y. Wan, Entanglement Entropy of Topological Orders with Boundaries, JHEP 06 (2018) 113 [arXiv:1804.05725] [INSPIRE].

[4] B. Shi, Seeing topological entanglement through the information convex, Phys. Rev. Research. 1 (2019) 033048 [arXiv: 1810.01986] [INSPIRE].

[5] J. Lou, C. Shen and L.-Y. Hung, Ishibashi states, topological orders with boundaries and topological entanglement entropy. Part I, JHEP 04 (2019) 017 [arXiv: 1901.08238] [INSPIRE].

[6] Y. Hu and Y. Wan, Entanglement Entropy, Quantum Fluctuations and Thermal Entropy in Topological Phases, JHEP 05 (2019) 110 [arXiv:1901.09033] [INSPIRE].

[7] Z.-X. Luo, B.G. Pankovich, Y. Hu and Y.-S. Wu, Correspondence between bulk entanglement and boundary excitation spectra in two-dimensional gapped topological phases, Phys. Rev. B 99 (2019) 205137 [arXiv: 1806.07794] [INSPIRE].

[8] S. Beigi, P.W. Shor and D. Whalen, The Quantum Double Model with Boundary: Condensations and Symmetries, Commun. Math. Phys. 306 (2011) 663.

[9] I. Cong, M. Cheng and Z. Wang, Topological Quantum Computation with Gapped Boundaries, arXiv:1609.02037.

[10] Y. Hu, Z.-X. Luo, R. Pankovich, Y. Wan and Y.-S. Wu, Boundary Hamiltonian theory for gapped topological phases on an open surface, JHEP 01 (2018) 134 [arXiv:1706.03329] [INSPIRE].

[11] Y. Hu, Y. Wan and Y.-S. Wu, From effective Hamiltonian to anomaly inflow in topological orders with boundaries, JHEP 08 (2018) 092 [arXiv:1706.09782] [INSPIRE].

[12] H. Wang, Y. Li, Y. Hu and Y. Wan, Gapped Boundary Theory of the Twisted Gauge Theory Model of Three-Dimensional Topological Orders, JHEP 10 (2018) 114 [arXiv:1807.11083] [INSPIRE].

[13] V.B. Petkova and J.B. Zuber, Generalized twisted partition functions, Phys. Lett. B 504 (2001) 157 [hep-th/0011021] [INSPIRE].

[14] C. Shen and L.-Y. Hung, Defect Verlinde Formula for Edge Excitations in Topological Order, Phys. Rev. Lett. 123 (2019) 051602 [arXiv: 1901.08285] [INSPIRE].

[15] S. Dong, E. Fradkin, R.G. Leigh and S. Nowling, Topological Entanglement Entropy in Chern-Simons Theories and Quantum Hall Fluids, JHEP 05 (2008) 016 [arXiv:0802.3231] [INSPIRE].

[16] J.R. Fliss et al., Interface Contributions to Topological Entanglement in Abelian Chern-Simons Theory, JHEP 09 (2017) 056 [arXiv: 1705.09611] [INSPIRE].

[17] X. Wen, S. Matsuura and S. Ryu, Edge theory approach to topological entanglement entropy, mutual information and entanglement negativity in Chern-Simons theories, Phys. Rev. B 93 (2016) 245140 [arXiv: 1603.08534] [INSPIRE].

[18] M. Barkeshli, C.-M. Jian and X.-L. Qi, Theory of defects in Abelian topological states, Phys. Rev. B 88 (2013) 235103 [arXiv: 1305.7203] [INSPIRE].

[19] T. Lan, J.C. Wang and X.-G. Wen, Gapped Domain Walls, Gapped Boundaries and Topological Degeneracy, Phys. Rev. Lett. 114 (2015) 076402 [arXiv:1408.6514] [InSPIRE].

[20] R. Blumenhagen and E. Plauschinn, Introduction to conformal field theory, Lect. Notes Phys. 779 (2009) 1 [INSPIRE]. 
[21] A. Kapustin and N. Saulina, Topological boundary conditions in abelian Chern-Simons theory, Nucl. Phys. B 845 (2011) 393 [arXiv: 1008.0654] [InSPIRE].

[22] M. Levin, Protected edge modes without symmetry, Phys. Rev. X 3 (2013) 021009 [arXiv:1301.7355] [INSPIRE].

[23] J.C. Wang and X.-G. Wen, Boundary Degeneracy of Topological Order, Phys. Rev. B 91 (2015) 125124 [arXiv:1212.4863] [INSPIRE].

[24] X.-G. Wen, Topological orders and edge excitations in FQH states, Adv. Phys. 44 (1995) 405 [cond-mat/9506066] [INSPIRE].

[25] F.A. Bais, B.J. Schroers and J.K. Slingerland, Hopf symmetry breaking and confinement in $(2+1)$-dimensional gauge theory, JHEP 05 (2003) 068 [hep-th/0205114] [INSPIRE].

[26] F.A. Bais, B.J. Schroers and J.K. Slingerland, Broken quantum symmetry and confinement phases in planar physics, Phys. Rev. Lett. 89 (2002) 181601 [hep-th/0205117] [INSPIRE].

[27] F.A. Bais and J.K. Slingerland, Condensate induced transitions between topologically ordered phases, Phys. Rev. B 79 (2009) 045316 [arXiv:0808.0627] [INSPIRE].

[28] F.A. Bais, J.K. Slingerland and S.M. Haaker, A Theory of topological edges and domain walls, Phys. Rev. Lett. 102 (2009) 220403 [arXiv:0812.4596] [INSPIRE].

[29] M. Barkeshli, C.-M. Jian and X.-L. Qi, Classification of Topological Defects in Abelian Topological States, Phys. Rev. B 88 (2013) 241103 [arXiv:1304.7579] [INSPIRE].

[30] L. Kong, Anyon condensation and tensor categories, Nucl. Phys. B 886 (2014) 436 [arXiv: 1307.8244] [INSPIRE].

[31] J. Fuchs, C. Schweigert and A. Valentino, Bicategories for boundary conditions and for surface defects in 3D TFT, Commun. Math. Phys. 321 (2013) 543 [arXiv:1203.4568] [INSPIRE].

[32] L.-Y. Hung and Y. Wan, Ground State Degeneracy of Topological Phases on Open Surfaces, Phys. Rev. Lett. 114 (2015) 076401 [arXiv: 1408.0014] [INSPIRE].

[33] L.-Y. Hung and Y. Wan, Generalized ADE classification of topological boundaries and anyon condensation, JHEP 07 (2015) 120 [arXiv: 1502.02026] [INSPIRE].

[34] J.L. Cardy, Boundary Conditions, Fusion Rules and the Verlinde Formula, Nucl. Phys. B 324 (1989) 581 [INSPIRE].

[35] J.L. Cardy, Boundary conformal field theory, hep-th/0411189 [INSPIRE].

[36] J.P. Serre, Cours d'arithmetique, Presses Universitaires De France (1994). 